\title{
Superrotation in Planetary Atmospheres
}

\author{
Takeshi Imamura ${ }^{1}$ Jonathan Mitchell ${ }^{2}$. \\ Sebastien Lebonnois ${ }^{3}$. Yohai Kaspi ${ }^{4}$. \\ Adam P. Showman ${ }^{5}$ Oleg Korablev ${ }^{6}$
}

Received: 12 November 2019 / Accepted: 6 June 2020 / Published online: 1 July 2020

(C) The Author(s) 2020

\begin{abstract}
Superrotation is a dynamical regime where the atmosphere circulates around the planet in the direction of planetary rotation with excess angular momentum in the equatorial region. Superrotation is known to exist in the atmospheres of Venus, Titan, Jupiter, and Saturn in the solar system. Some of the exoplanets also exhibit superrotation. Our understanding of superrotation in a framework of circulation regimes of the atmospheres of terrestrial planets is in progress thanks to the development of numerical models; a global instability involving planetary-scale waves seems to play a key role, and the dynamical state depends on the Rossby number, a measure of the relative importance of the inertial and Coriolis forces, and the thermal inertia of the atmosphere. Recent general circulation models of Venus's and Titan's atmospheres demonstrated the importance of horizontal waves in the angular momentum transport in these atmospheres and also an additional contribution of thermal tides in Venus's atmosphere. The atmospheres of Jupiter and Saturn also exhibit strong superrotation. Recent gravity data suggests that these superrotational flows extend deep into the planet, yet currently no single mechanism has been identified as driving this superrotation. Moreover, atmospheric circulation models of tidally locked, strongly
\end{abstract}

Understanding the Diversity of Planetary Atmospheres

Edited by François Forget, Oleg Korablev, Julia Venturini, Takeshi Imamura, Helmut Lammer and Michel Blanc

T. Imamura

t_imamura@edu.k.u-tokyo.ac.jp

1 Graduate School of Frontier Sciences, The University of Tokyo, Kashiwa, Japan

2 Department of Earth, Planetary and Space Sciences, Department of Atmospheric and Oceanic Sciences, University of California, Los Angeles, CA, USA

3 Laboratoire de Météorologie Dynamique (LMD)/IPSL, Sorbonne University, ENS, PSL Research University, Institut Polytechnique de Paris, CNRS, Paris, France

4 Department of Earth and Planetary Sciences, Weizmann Institute of Science, Rehovot 76100, Israel

5 Lunar and Planetary Laboratory, Department of Planetary Sciences, The University of Arizona, Tucson, AZ 85721, USA

6 Space Research Institute (IKI), Russian Academy of Sciences, 84/32 Profsoyuznaya, 117997 Moscow, Russia 
irradiated exoplanets have long predicted the existence of equatorial superrotation in their atmospheres, which has been attributed to the result of the strong day-night thermal forcing. As predicted, recent Doppler observations and infrared phase curves of hot Jupiters appear to confirm the presence of superrotation on these objects.

Keywords Superrotation · Planetary atmosphere $\cdot$ Venus · Titan · Gas giants · Exoplanets

\section{Introduction}

\subsection{Superrotation on Various Celestial Bodies}

Superrotation is a dynamical regime where the atmosphere moves around the planet in the direction of planetary rotation, and is usually defined as the atmospheric angular velocity in the equatorial region exceeding that of the solid planet. Superrotation permanently exists in the atmospheres of Venus, Titan, Jupiter and Saturn (Read and Lebonnois 2018) (Fig. 1). Though Earth's equatorial atmosphere is subrotating on average in the troposphere, the equatorial stratosphere exhibits superrotation locally and temporarily as a part of the quasi-biennial oscillation (Baldwin et al. 2001). The wind distribution of Mars undergoes large seasonal variation and does not show a permanent superrotation. Moreover, many exoplanets are thought to have superrotating atmospheres (Showman et al. 2010, 2013a, 2013b; Heng and Showman 2015). The basic parameters of the known superrotating atmospheres are summarized in Table 1. Understanding of superrotation in a framework of circulation regimes of planetary atmospheres is thought to be crucial to the understanding of the diversity of the atmospheric environment in and out of the solar system. Observations of key processes driving superrotation are ongoing especially for Venus' atmosphere. This article reviews the current knowledge on superrotation and prospects for future research.

The most prominent superrotation is found in the atmosphere of Venus (Sánchez-Lavega et al. 2017). According to remote and direct measurements, the atmosphere at heights of 60-70 km above the surface travels around the planet in 4-5 Earth days, corresponding to speeds of $\sim 100 \mathrm{~m} \mathrm{~s}^{-1}$. This corresponds to an angular velocity about the rotation axis that is more than 50 times faster than the rotation rate of the solid planet. Below this height, the velocity decreases monotonically with decreasing altitude, and it becomes $<1 \mathrm{~m} \mathrm{~s}^{-1}$ near the surface (Schubert et al. 1980). Because of the fast rotation, the temperature and clouds in the middle atmosphere $(>50 \mathrm{~km})$ are zonally well smoothed despite the fact that the radiative relaxation time in this height region is comparable to or shorter than the length of a solar day of 117 Earth days (Pollack and Young 1975). Since the discovery of superrotation on Venus in the 1960s, its mechanism has been a major issue in the fluid dynamics of planetary atmospheres. Superrotation occurs also in the atmosphere of Titan, one of Saturn's satellites. Titan's large stratospheric zonal winds on the order of $100 \mathrm{~m} \mathrm{~s}^{-1}$ have been inferred from remote observations and were confirmed by a descent probe (Bird et al. 2005). Given the slow rotation of the satellite with a period of 16 Earth days, the superrotation is about 10 times faster than the rotation of the solid surface. The mechanisms of the extreme superrotation on Venus and Titan are not well understood and pose difficult challenges for numerical models (Read and Lebonnois 2018).

Jupiter and Saturn also exhibit strong superrotation in the equatorial region at the level of the visible cloud tops. A striking difference from the terrestrial planets is that Jupiter and Saturn have an internal heat flux (Hanel et al. 1983; Li et al. 2018), which possibly drives the deep circulations. The wind speeds of the eastward zonal jets reach $\sim 100$ and $\sim 400 \mathrm{~m} \mathrm{~s}^{-1}$, 


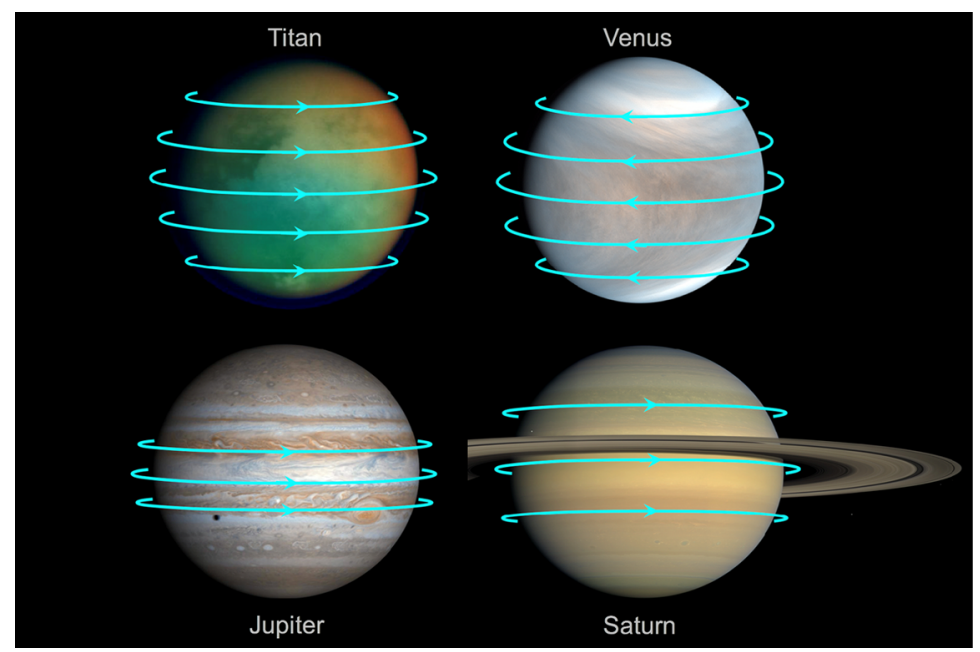

Fig. 1 Schematic of the direction and the latitudinal extent of superrotation for Titan, Venus, Jupiter and Saturn. Superrotation extends to the high latitudes on Titan and Venus, while it is confined to the low latitudes on Jupiter and Saturn $\left(<15^{\circ}\right.$ for Jupiter and $<30^{\circ}$ for Saturn). The superrotation of Venus is westward, while those of the others are eastward (but note that in all cases, superrotation implies prograde flow, that is, flow relative to the planet's surface in the same direction as the planetary rotation) (The images of Titan, Jupiter and Saturn were provided by NASA. The Venus image was provided by JAXA)

Table 1 Basic information on the superrotation of the atmospheres of the solar system planets and a hot Jupiter (modified from Read and Lebonnois 2018)

\begin{tabular}{llllll}
\hline Planet & $\begin{array}{l}\text { Radius } \\
(\mathrm{km})\end{array}$ & $\begin{array}{l}\text { Rotation } \\
\text { period (days) }\end{array}$ & $\begin{array}{l}\text { Equatorial rotation } \\
\text { speed }(\mathrm{m} / \mathrm{s})\end{array}$ & $\begin{array}{l}\text { Equatorial wind } \\
\text { speed }(\mathrm{m} / \mathrm{s})\end{array}$ & $\begin{array}{l}\text { Superrotation index, } \\
\text { s, on the equator }\end{array}$ \\
\hline Venus & 6,052 & 243 & 1.81 & $100-120$ & $55-66$ \\
Titan & 2,576 & 16.0 & 11.7 & $100-180$ & $8.5-15$ \\
Jupiter & 69,911 & 0.41 & 12,300 & $60-140$ & $0.005-0.011$ \\
Saturn & 58,232 & 0.44 & 9,540 & $350-430$ & $0.037-0.045$ \\
HD 189733b & 79,500 & 2.2 & 2600 & 2400 & 0.92 \\
\hline
\end{tabular}

respectively, on Jupiter and Saturn (Simon et al. 2015; Choi et al. 2009). The vertical structures of the flows below the cloud tops have long been unknown. Previous investigations on the flow pattern are broadly categorized into two types: shallow terrestrial-like models, in which motions are restricted to the thin layer near the cloud top in which the flows are driven by geostrophic turbulence, and deep models, in which deep flows are driven by convection transporting heat upward throughout the planetary interior. The observational landscape is changing rapidly, as gravity measurements from NASA's Juno mission to Jupiter and Cassini Grand Finale to Saturn have recently been obtained; these datasets provide information on the mass distribution and flow profile in the deep atmospheres of Jupiter and Saturn (Kaspi et al. 2018, 2020; Galanti et al. 2019).

Theories and models suggest that tidally locked exoplanets can exhibit circulations dominated by equatorial superrotation (Joshi et al. 1997; Showman and Guillot 2002; Showman and Polvani 2011). Showman and Guillot (2002) predicted that strong superrotation occurring on close-in, strongly irradiated giant exoplanets- the "hot Jupiters"-would 
cause an eastward displacement of the dayside hot spot on these worlds. Such eastward displacements of the infrared (thermal) hot spots were first confirmed for the hot Jupiter HD 189733 b by Knutson et al. (2007) and have now been observed for a wide range of hot Jupiters (see Heng and Showman 2015 for a review). Such observational constraints are harder to obtain for smaller planets, but recently, thermal infrared phase curve observations for a super Earth exoplanet have similarly shown such an eastward displacement of the dayside hot spot from the substellar point (Demory et al. 2016). Moreover, direct observational confirmation of equatorial superrotation on the hot Jupiter HD 189733b has been obtained by observing the Doppler shift separately on the planet's leading and trailing limbs as it transits its star (Louden and Wheatley 2015). These Doppler observations indicate superrotating wind speeds of several $\mathrm{km} / \mathrm{s}$, generally consistent with predictions from general circulation models (GCMs) (e.g., Kempton and Rauscher 2012; Showman et al. 2013b; Flowers et al. 2019). Understanding of circulation regimes should cover such tidally locked planets as well. Strong zonal flows are expected to transport heat from the permanentlyilluminated side to the perpetually-dark side, and on terrestrial exoplanets might contribute to formation of a habitable environment in some conditions by relaxing the day-night temperature contrast.

The internal rotation profile of the Sun inferred from helioseismology shows a prominent enhancement of the angular velocity in the low latitudes (Schou et al. 1998), which can also be considered as equatorial superrotation. The angular velocity decreases monotonically from the equator to the pole by about $30 \%$ throughout the convective envelope. A deviation from a cylindrical profile expected from the Taylor-Proudman theorem is observed. Considering that the fluid dynamics of the Sun are driven by internal heat flux similar to those of Jupiter and Saturn, a comparative study of the Sun and the gas giants could yield insights. Equatorward angular momentum transport by tilted columnar convection is considered to maintain the superrotation (Miesch et al. 2006).

A quantitative measure of superrotation can be a relative excess of angular momentum compared with the value it would take in co-rotation with the underlying solid planet or the bulk of the planet (Read 1986):

$$
s=\frac{m}{\Omega a^{2}}-1,
$$

where $\Omega$ is the angular velocity of planetary rotation, $a$ is the planetary radius, and $m$ is the axial component of specific absolute angular momentum defined by

$$
m=a \cos \phi(\Omega a \cos \phi+u),
$$

with $\phi$ being the latitude and $u$ the zonal velocity. The local superrotation is defined by $s>0$. A comprehensive comparison among the planets in terms of the index $s$ in the equatorial region is given in Read and Lebonnois (2018): Venus exhibits the greatest value of $s=55-65$ around $70 \mathrm{~km}$ altitude, Titan has $s=8.5-15$ above $100 \mathrm{~km}$ altitude, Jupiter has $s=0.005-0.011$ at the visible cloud top, Saturn has $s=0.035-0.045$ at the visible cloud top, and the hot Jupiter HD 189733b seems to have $s \sim 1$. The key physical processes that control the index $s$ are hardly identified. Another index of superrotation representing a global excess angular momentum has also been proposed (Read 1986; Read and Lebonnois 2018). Equivalently, an angular momentum conserving wind can be defined by conservation of angular momentum $(m)$ of an air parcel at latitude $\phi$ that moves poleward from rest at the equator:

$$
u_{M}=\Omega a \frac{\sin ^{2} \phi}{\cos \phi} .
$$


Fig. 2 Schematic of cyclostrophic balance, where the centrifugal force balances the horizontal pressure gradient force

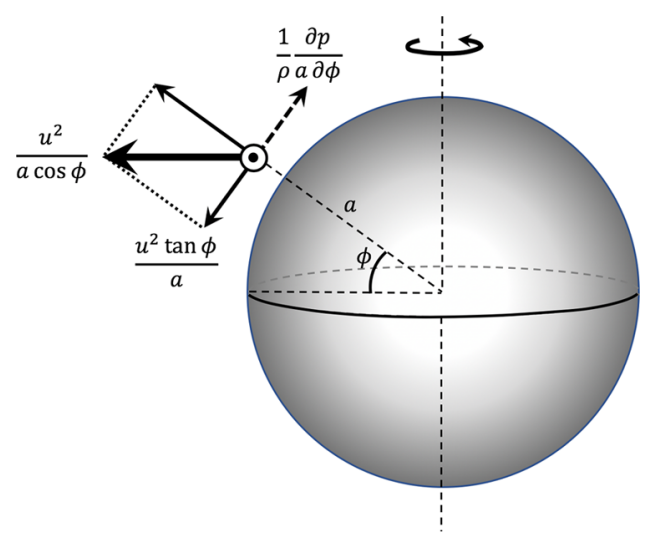

Any wind velocity faster than $u_{M}$ is superrotating. As this function depends strongly on latitude it is obvious that only low latitude winds can be superrotating.

\subsection{Dynamical Structure and Eddy Momentum Transport}

On rapidly rotating planets such as Earth, Jupiter and Saturn, Coriolis acceleration roughly balances the horizontal pressure gradient in the meridional momentum equation. On the other hand, the dynamical balance of slowly-rotating planets having superrotating atmospheres, such as Venus and Titan, is thought to be "cyclostrophic," where centrifugal acceleration balances the horizontal pressure gradient (e.g., Sánchez-Lavega et al. 2017):

$$
u^{2} \tan \phi+\frac{1}{\rho} \frac{\partial p}{\partial \phi}=0
$$

where $\rho$ is the atmospheric density and $p$ is the pressure (Fig. 2). By combining the equation above with the equation of hydrostatic equilibrium and the ideal gas law, we further obtain the following thermal wind equation in pressure coordinates:

$$
\frac{\partial u^{2}}{\partial \zeta}+\frac{R}{\tan \phi} \frac{\partial T}{\partial \phi}=0,
$$

where $\zeta=-\ln \left(p / p_{0}\right)$ with $p_{0}$ being a reference pressure, $T$ is the temperature and $R$ is the gas constant. Given a latitudinal profile of $u$ at a particular pressure level and measurements of $T$ as a function of height and latitude, this equation can be integrated vertically to retrieve the zonal velocity field $u(\phi, p)$. This method has been used for Venus and Titan (Piccialli et al. 2012; Achterberg et al. 2008). The velocity field of Venus inferred from temperature measurements is roughly consistent with the cloud-tracked velocities at the cloud top level, although some discrepancies still need to be explained (Newman et al. 1984; Piccialli et al. 2012).

The specific angular momentum $m$ is conserved following motions in the meridional plane in an axisymmetric flow in the absence of external torques (Held and Hou 1980). This principle leads to an argument that axisymmetric circulation cannot maintain a local maximum in the angular momentum above the equator: angular-momentum-conserving meridional circulation transports air having smaller angular momentum to the equatorial region and tends to decelerate equatorial superrotation. If we reasonably assume that eddy viscous diffusion is always downgradient, the superrotation should eventually disappear. Hide 
(1969) suggested that non-axisymmetric eddies that involve local azimuthal pressure gradients are needed to maintain the jets and that the required momentum convergence should be provided by upgradient angular momentum transport. Eddies contributing to such angular momentum transport are thought to be mostly atmospheric waves, and considered to be different among the planets; possible scenarios will be presented in later sections.

The physical processes that determine the height to which a superrotational flow can extend are not well understood. The higher the altitude, the shorter the radiative relaxation time and the greater the day-night temperature difference. Such a strong thermal forcing can induce subsolar-to-antisolar (SS-AS) circulation instead of superrotation (Seiff 1982; Gorinov et al. 2018). On the other hand, a strong thermal forcing can induce eddy momentum fluxes via generation of solar-locked waves (tides), which potentially induces equatorial superrotation (Takagi and Matsuda 2007; Showman and Polvani 2011); evaluation of competing processes is crucial. Furthermore, effects of momentum transport by upwardlypropagating waves can become greater at higher altitudes because of the amplitude growth with height (Fritts and Alexander 2003). The large molecular viscosity coefficient at high altitudes should also influence the circulation. Considering that information on the atmospheres of exoplanets obtained by transit spectroscopy tends to be limited to the upper atmosphere, the understanding of physical processes determining the vertical extent of superrotation is crucial especially for exoplanet researches. In Venus' thermosphere, SS-AS circulation was found to be comparable in magnitude with the superrotation (Clancy et al. 2012).

\subsection{Circulation Regimes}

The principle of dynamic similarity - (stated loosely) that fluids with similar nondimensional combinations of dimensional parameters behave similarly - allows one to study the dynamics of atmospheric circulations by smoothly varying a small number of control parameters, for instance such that one passes from an Earth-like regime to a Titan-like one by varying a single dimensional parameter. This approach often employs idealized and/or analog frameworks that may, for instance, remove all continents and oceans, the condensable component of the atmosphere, simplify the heating that drives the circulation, and perhaps even change the geometry of the fluid basin. As drastic as these simplifications may sound, there is a rich history studying simplified, analog systems of equations in geophysical fluid dynamics (e.g. Held and Hou 1980; Williams 1988). Once simplified, these sets of governing equations lend themselves to nondimensionalization of, for instance, the component equations of motion. The Rossby number is perhaps the best example of a non-dimensional coefficient of the inertial term in the nondimensionalized equations of motion, and it is a measure of the relative importance of the inertial and Coriolis forces. Earth, Mars, Jupiter and Saturn all have small Rossby numbers, meaning that rotation strongly constrains fluid motions. Venus and Titan, on the other hand, have large Rossby numbers, and rotation is less important (but not negligible). It is likely not a coincidence that the two terrestrial bodies with large Rossby numbers have superrotating atmospheres.

Other non-dimensional coefficients include the Ekman number, Rayleigh number, Prandtl number, etc. By quantifying these non-dimensional numbers, the individual planetary objects of the Solar System and exoplanets with their discrete parameters are simply particular realizations of the broader, continuous parameter space of planetary climate represented by these non-dimensional numbers. An advantage of using this approach is that the fundamental dynamics of certain classes of phenomena can be easily identified and studied; atmospheric superrotation is a very good example. 
A number of authors have used the dynamical similarity approach to understanding atmospheric superrotation (e.g., Williams 1988; Read 2011; Mitchell and Vallis 2010; Potter et al. 2014). These share in common the construction of "numerical experiments" using simplified versions of GCMs with the moist thermodynamics, radiative transfer and complicated boundary conditions stripped away. The GCMs are driven by a simple linear relaxation to a specified temperature structure, typically taken to be static and hemispherically and zonally symmetric. The experiment design often involves the adjustment of a single or perhaps two parameters, and the focus is on finding the transition state from sub- to super-rotating winds. Mitchell and Vallis (2010) captured the transition to superrotation by increasing the Rossby number through shrinking the planetary size; interestingly, the same transition does not occur if the rotation rate is decreased instead of the planetary size, an effect most likely resulting from a strong Hadley circulation (dias Pinto and Mitchell 2014; Guendelman and Kaspi 2018, 2019). The transition itself occurs somewhere between the Rossby numbers for Earth and Titan, but for which we have no observable analog. By studying the intermediate, transitional state, Potter et al. (2014) clearly demonstrated a role for equatorial Kelvin waves in accelerating the superrotating equatorial winds. This is somewhat of a surprise, given a pure Kelvin wave on an equatorial beta plane has no potential vorticity anomaly, and also no meridional flow if there is no background flow (e.g., Holton and Hakim 2013; Andrews et al. 1987). This would imply that Kelvin waves do not meridionally transport momentum, although on a sphere the structure of a Kelvin wave changes (Garfinkel et al. 2017; Yamamoto 2019). However, in a series of linear instability calculations, Wang and Mitchell (2014) demonstrated a global "ageostrophic instability" involving a coupling of Kelvin and Rossby waves is responsible for the transition to superrotation seen in idealized GCM experiments. A feature like this had been identified in previous, pioneering work in channelized shallow-water on the equatorial plane (Sakai 1989).

Figure 3 displays the phenomenology of the Rossby-Kelvin instability, both in idealized GCM simulations and in linear eigenvalue calculations. The Kelvin wave straddles the equator and the Rossby waves are confined by mid- to high-latitude jet streams. Instability, and ultimately a transition to the superrotating state, requires there to be overlap between the Rossby and Kelvin waves and for them to lock phase with one another. Note that another wave mode involving only Rossby waves is likely responsible for the maintenance of superrotation in the high-Rossby-number regime (dias Pinto and Mitchell 2014).

The relatively simple picture of global ageostrophic instability leading to superrotation in the appropriate parameter regime is complicated by many factors, not least of which is the presence of a seasonal cycle. The easterly torque provided by a cross-equatorial Hadley circulation can be enough to prevent the spinup and maintenance of equatorial superrotation (Mitchell et al. 2014). However, it would seem that a simple slowing down of the Hadley circulation is all that is needed to restore superrotation in the presence of a strong seasonal cycle; this can be accomplished, for instance, by dramatically increasing the time it takes the atmosphere to radiatively cool, thereby increasing the thermal inertia of the atmosphere. Something like this may indeed be happening in Titan's stratosphere, where strong superrotation exists in the mean, but varies in magnitude as the Hadley circulation develops crossequatorial flow (Newman et al. 2011; also see Sect. 2.2). And although Venus does not have a significant seasonal cycle, its massive atmosphere and thermal inertia has the same effect of slowing down the Hadley cell, allowing strong superrotation to be sustained.

An intermediate approach between GCM simulations and linear eigenvalue solvers is to simplify the governing equations to two-dimensional zonal mean and parameterize the influence of three-dimensional eddies on heat and momentum. In this approach, it is common to approximate eddy momentum transport as a momentum diffusion (e.g. Yamamoto and Yoden 2013), an idea first put forward by Gierasch (1975) to explain superrotation on Venus. 
Fig. 3 (a) The longitude-latitude pattern for pressure perturbation (red represents positive, blue represents negative) and wind anomalies (vectors) of an unstable Rossby-Kelvin mode from linear analysis, and the associated mean background meridional shear $U(\varphi)$ (white line, scaled by $U_{0}$ ). (b) The potential vorticity gradient $P V_{\varphi}$ of the mean shear used in our linear analysis (scaled by $2 \Omega$ ).

(c) The zonal momentum acceleration of the linear, unstable mode (Fig. 3a) as a function of latitude (scaled by $4 a \Omega^{2}$ ). (d) The $700 \mathrm{hPa}$ geopotential height perturbation (color) and wind anomalies (vectors) of the mode accelerating the equatorial zonal winds, and vertical and zonal mean zonal wind (white line) (both) from the Titan-like simulation of Mitchell and Vallis (2010). Reproduced from Wang and Mitchell (2014)
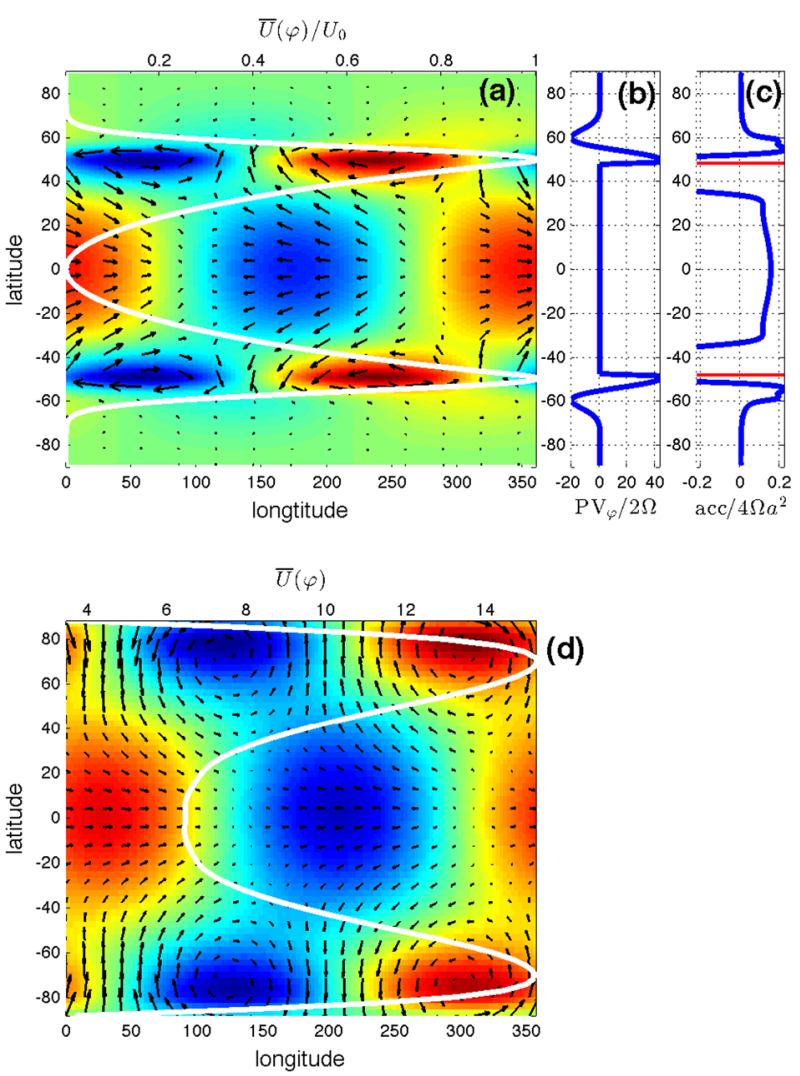

As with GCM simulations, this type of parameterized model can be used to construct regime diagrams of atmospheric circulation based on a set of non-dimensional control parameters (Matsuda 1980; Kashimura and Yoden 2015).

\section{Superrotation on Venus and Titan}

\subsection{Venus}

\subsubsection{Observations of Venus's Atmospheric Circulation}

Venus's atmosphere is characterized by a thick cloud layer completely covering the planet, at altitudes between $\sim 48$ and $\sim 70 \mathrm{~km}$. Thanks to a mysterious and yet unidentified ultraviolet absorber located in the upper cloud layers (roughly 60-70 km), sharp contrasts can be observed from space. When they are moving with the flow, these contrasts are used to measure the wind speed in the layers they are embedded in. Depending on the wavelength, these cloud-tracking winds are representative of different atmospheric levels, from $\sim 70 \mathrm{~km}$ in UV to $\sim 60 \mathrm{~km}$ in near infrared. For these wavelengths, winds can be measured only on the day side, but the same technique can also be used at other wavelengths. In the thermal infrared (e.g. around 5 microns), contrasts are also observed in the brightness temperature, allowing the determination of wind speed near the cloud top 
Fig. 4 Zonal wind velocity profiles acquired by Venera and Pioneer Venus probes as summarized by Schubert et al. (1980)

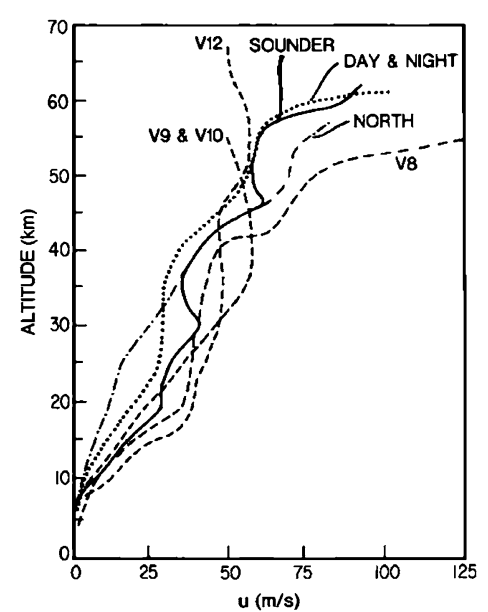

both on day and night sides. On the night side, in some infrared windows (e.g. at 1.74 or 2.3 microns), emissions formed below the clouds are observed from space, and contrasts due to variations in the lower cloud opacity can be followed to measure winds near $50 \mathrm{~km}$ altitude. Therefore, these cloud-tracking winds give us access to maps of the circulation, for several levels in the cloud layers (e.g. Limaye and Suomi 1981; Limaye 2007; Sánchez-Lavega et al. 2008; Khatuntsev et al. 2013, 2017; Kouyama et al. 2013; Horinouchi et al. 2017, 2018).

Tracking in-situ probes was a technique also used for the Venera (Kerzhanovich and Marov 1983), Pioneer Venus (Counselman et al. 1980), and VeGa (Moroz and Zasova 1997) probes. This gave access to vertical profiles of the wind from roughly $60 \mathrm{~km}$ altitude to the surface (Fig. 4). These are for the moment the only measurements of the wind speeds for altitudes below the cloud base. The VeGa balloons were also tracked during their flights in the $54 \mathrm{~km}$ altitude layer (Linkin et al. 1986) providing a direct evidence of the superrotation.

Temperature maps obtained from various spacecraft instruments (radio occultation, infrared spectrometers and spectro-imagers) can also be used to retrieve zonal wind fields assuming cyclostrophic balance (e.g., Zasova et al. 2000; Piccialli et al. 2008, 2012), although this is an indirect method. This method gives access to zonal wind maps in the $45-95 \mathrm{~km}$ altitude range.

Gorinov et al. (2018) used tracking of $\mathrm{O}_{2}(\mathrm{a} 1 \Delta \mathrm{g}) 1.27 \mu \mathrm{m}$ nightglow from VIRTIS onboard Venus Express, which corresponds to altitudes of $90-110 \mathrm{~km}$, the region in between the superrotation and the SS-AS circulation. Two opposite flows from the terminator to midnight were observed. The wind from the morning side exceeds the evening one by 20 $30 \mathrm{~m} / \mathrm{s}$, and the two streams meet at $\sim 22.5 \mathrm{~h}$.

From Earth, observations of the Doppler shift of different types of spectral lines allow the determination of line-of-sight wind speed. Fraunhofer solar reflected lines in the visible and near-infrared are used to probe the cloud-top region and show a good agreement with cloudtracking measurements (Machado et al. 2014, 2017). $\mathrm{CO}_{2}$ absorption lines in millimeter or sub-millimeter wavelengths probe higher in the mesosphere $(90-105 \mathrm{~km})$, as well as $\mathrm{CO}_{2}$ non-LTE emissions at 5 and 10 microns $(\sim 110 \mathrm{~km})$.

An example of the zonal and meridional wind fields measured by cloud-tracking at $365 \mathrm{~nm}$ with the UVI instrument on-board the Akatsuki spacecraft is shown in Fig. 5. At the cloud top, zonal winds show superrotation, with velocities around 100-120 m/s (50 to 

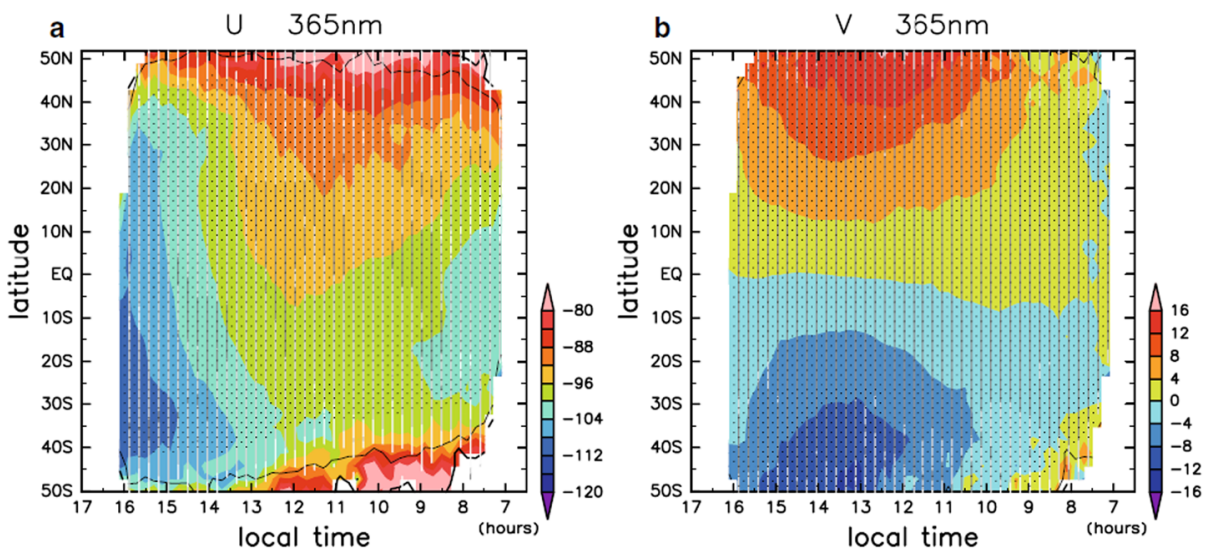

Fig. 5 Mean winds obtained from clouds observed at $365 \mathrm{~nm}$ during the period from December 2015 to March 2017 with the UVI/Akatsuki camera, as a function of local time and latitude. (a) Zonal wind (m/s); (b) Meridional wind (m/s). From Horinouchi et al. (2018)

60 times the surface rotation speed, as the rotation period is 243 Earth days) in the equatorial to mid-latitude regions. Velocities decrease then almost linearly with latitude down to zero at the pole. In the clouds, a strong wind shear is measured in the equatorial to midlatitude regions, with zonal wind decreasing to $\sim 60 \mathrm{~m} / \mathrm{s}$ near the cloud base, while in the polar regions, the zonal wind appears to be much more uniform in the vertical direction. Though limited to a few locations and local times, the vertical profiles measured by the different descending probes show that the zonal wind decreases everywhere with decreasing altitude, from the cloud top to roughly $10 \mathrm{~km}$ altitude, below which the winds are quite small (below a couple of $\mathrm{m} / \mathrm{s}$ ). Some latitudinal variations are observed in the deep atmosphere, below the clouds. Variability as a function of local time has been observed in the cloud region, reflecting the impact of thermal tides on the wind field (e.g. Horinouchi et al. 2018; Patsaeva et al. 2019). At cloud top, indications of long-term variations have been noted (Khatuntsev et al. 2013; Horinouchi et al. 2018), although the observed trend might be influenced by the dependence on the surface topography (Bertaux et al. 2016; Khatuntsev et al. 2017). Short-term variability has been identified in the zonal and meridional winds and cloud brightness fields, revealing the presence in the upper cloud of wavenumber-1 Kelvin and Rossby type waves with periods of the order of 4 to 5 days (e.g. Del Genio and Rossow 1990; Rossow et al. 1990; Peralta et al. 2007, 2015; Kouyama et al. 2012; Imai et al. 2019). A recent review of the atmospheric dynamics of Venus may be found in Sánchez-Lavega et al. (2017).

\subsubsection{A Short History of Venus's General Circulation Models}

Though theoretical considerations are a useful guide, numerical atmospheric models are crucial tools to understand the superrotation mechanisms. Development of GCMs of Venus's atmosphere begin in the late 1970s, motivated by the space missions of this period. A recent review of the historical efforts done for the modeling of Venus's atmospheric circulation has been published by Lewis et al. (2013). Reproducing superrotation was difficult for the early GCMs, raising many questions (Young and Pollack 1977; Mayr and Harris 1983; Rossow 1983). Many improved GCMs with simplified radiative forcing were developed in the early 2000s (Yamamoto and Takahashi 2003, 2004, 2009; Lee et al. 2005, 2007; Lewis et al. 2006; 
Takagi and Matsuda 2007; Hollingsworth et al. 2007; Herrnstein and Dowling 2007; Lee and Richardson 2010), though they mostly developed superrotation only with an unrealistic solar forcing. These works obtained zonal wind maximum values near the cloud top, with high-latitude jets of various amplitudes, and a meridional stream function showing two large equator-to-pole circulation cells. Though the wave activity depends on the GCM, the roles of horizontal waves and thermal tides were studied in these different works, demonstrating the role they play in the angular momentum transport.

The most recent GCM including a simplified thermal forcing is based on the Atmospheric GCM For the Earth Simulator (AFES) and was developed by Sugimoto et al. (2014a, 2014b). The design of the thermal forcing includes a latitudinally dependent solar forcing and a Newtonian relaxation towards a latitudinally dependent temperature distribution, that includes a static stability profile designed to reproduce the convective layer in the cloud region. The spectral dynamical core is used at T42L60 or T63L120 resolution (where T is the truncation degree and $L$ the number of vertical levels) and extends up to $120 \mathrm{~km}$. The initial state was already in superrotation and maintained over 15 to 30 Venus days, with a zonal wind distribution that is very close to observations. The role of baroclinic instabilities in the upper cloud layers is emphasized in these results.

Full radiative transfer computations have been included in several recent GCMs (Ikeda 2011; Lebonnois et al. 2010, 2016; Mendonca and Read 2016; Yamamoto et al. 2019), with several different techniques. The IPSL Venus GCM (Lebonnois et al. 2010, 2016), developed at Institut Pierre-Simon Laplace, includes two innovative approaches: a Net Exchange Rate matrix is used to compute the energy exchanges between the different layers of the atmosphere in the infrared (Eymet et al. 2009), and the dynamical core takes into account the variation of the specific heat with temperature, which is significant in the case of the atmosphere of Venus and affects the definition of the potential temperature used in the dynamical core equations.

These most recent GCMs were more successful in the production and maintainance of superrotation. However, the different studies illustrated several weak points that need to be considered very carefully when modeling Venus's atmospheric superrotation: horizontal and vertical resolution, topography, angular momentum conservation (Lebonnois et al. 2012a), and vertical viscosity (Sugimoto et al. 2019a). The circulations modeled by the most recent GCMs agree in many aspects. The basic mechanism for developing and maintaining superrotation in Venus's atmosphere is a balance in the angular momentum transport between the mean meridional circulation and activity associated with various waves. This balance is extremely sensitive to many details in the models, as illustrated by the various simulations of the IPSL (Lebonnois et al. 2010, 2016; Garate-Lopez and Lebonnois 2018) or AFES (Sugimoto et al. 2014a, 2014b, 2019a) GCMs, and also by the intercomparison of several simple-forcing GCMs in the work by Lebonnois et al. (2013). This sensitivity reveals a very weakly-forced system.

\subsubsection{Current Interpretation of Venus's Superrotation}

The zonal wind fields obtained in recent GCMs is illustrated in Fig. 6. These distributions are not fully consistent with the observations, though some characteristics are well reproduced. The zonal wind peaks near the cloud top, with values consistent with observations. Highlatitude jets are present at latitudes too close to the poles in some simulations, e.g. for the IPSL Venus GCM. The vertical profile of the zonal wind is not always consistent with the observations. However, it is possible to draw some conclusions on the mechanisms that drive the superrotation on Venus. 

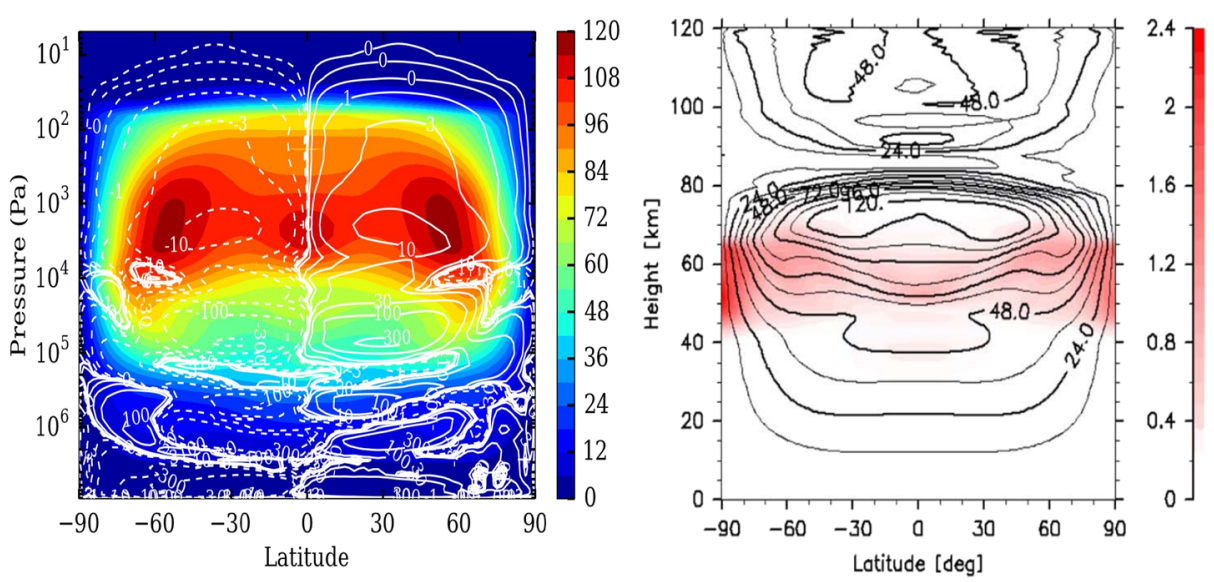

Fig. 6 Zonal wind distributions (left) in the IPSL Venus GCM (based on Garate-Lopez and Lebonnois 2018), in color (unit is $\mathrm{m} / \mathrm{s}$ ), with the mean meridional stream function as contours (unit is $10^{9} \mathrm{~kg} / \mathrm{s}$ ) and (right) in the AFES Venus GCM (from Sugimoto et al. 2014b), in contours, with the eddy kinetic wave activity in colors, defined as $\left(u^{\prime 2}+v^{\prime 2}\right) / 2$, where $u^{\prime}$ and $v^{\prime}$ are the eddy zonal and meridional velocities
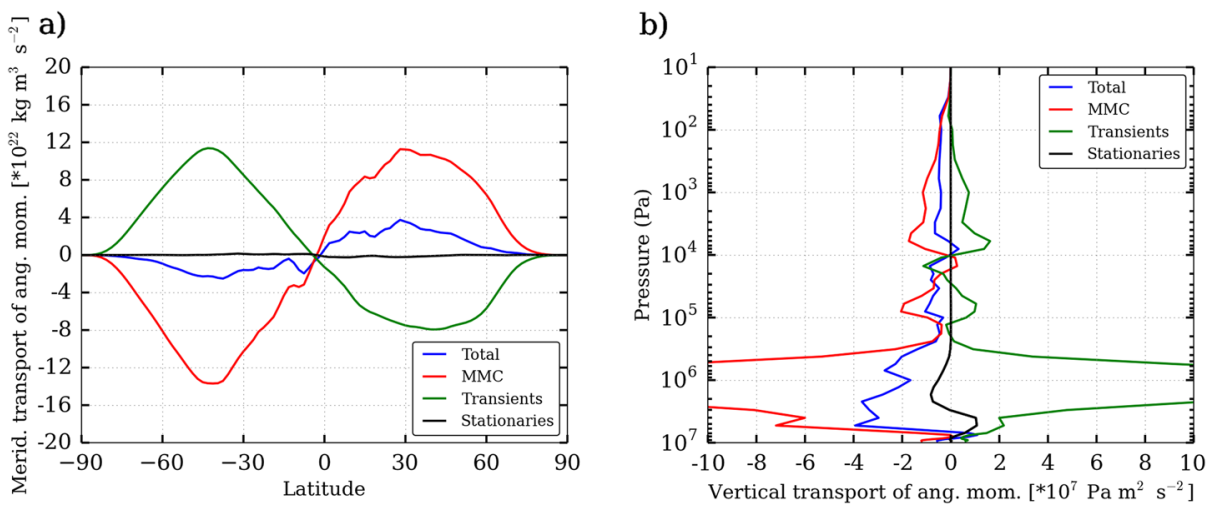

Fig. 7 Transport of angular momentum in the IPSL Venus GCM, with contributions of mean meridional circulation (MMC), planetary-scale waves (transients) and stationary waves (stationaries, related to the solid planet frame). (a) Vertically integrated meridional transport, positive is northward. (b) Horizontally integrated vertical transport, positive is downward. From Lebonnois et al. (2016)

The mean meridional circulation is illustrated in Fig. 6 in the case of the IPSL Venus GCM, with stacked equator-to-pole Hadley-type cells, in the middle cloud, one in the upper cloud and above, and one for the deep atmosphere, heavily perturbed by the impact of topography. This circulation transports angular momentum upward and poleward, and the budget is balanced by the eddy component of the angular momentum transport, as proposed in the Gierasch-Rossow-Williams mechanism (Gierasch 1975; Rossow and Williams 1979) and as illustrated in Fig. 7 in the case of the IPSL Venus GCM (Lebonnois et al. 2016).

The role of various types of wave activity is therefore crucial for the zonal wind distribution. In the upper cloud equatorial region, the thermal tides appear to play a significant role in many studies (Takagi and Matsuda 2007; Sugimoto et al. 2014a; Lebonnois et al. 2010; 
Takagi et al. 2018). As it was shown in Lebonnois et al. (2010), their impact on the angular momentum budget is visible in the transient term in Fig. $7 \mathrm{~b}$, above $10^{4} \mathrm{~Pa}$. A significant horizontal angular momentum transport is done by wave activity in the upper cloud region, related to barotropic instability near the poles and baroclinic instabilities at mid- to highlatitudes (Sugimoto et al. 2014b; Lebonnois et al. 2016), possibly related to the Rossby-type waves observed at the cloud top. It must be noted that these GCM simulations do not show Kelvin waves at the cloud top, though they do have Kelvin waves lower in the cloud layers. In the simulations published by Garate-Lopez and Lebonnois (2018), additional wave activity is obtained near the cloud base, that plays a significant role in improving the zonal wind profile around this region. Below the clouds, Lebonnois et al. (2016) report the presence in these simulations of large-scale gravity waves propagating downward and equatorward, resulting in a significant contribution to the deep atmosphere angular moment budget, increasing the zonal wind speeds in this region. All these large-scale wave activities are sensitive to modeling details, and comparison between several GCM results is needed to confirm their respective role in the atmosphere of Venus. In addition, the role of small-scale gravity waves, both of orographic and non-orographic origins, remain to be fully assessed.

The complete balance of the angular momentum budget in the atmosphere of Venus is therefore difficult to fully assess, as the respective role of each term, due to the sensitivity of the simulations to model details. The IPSL Venus GCM (Lebonnois et al. 2010, 2016) and AFES VGCM (Sugimoto et al. 2019a) have been able to build superrotation from a motionless state, demonstrating that the origin of the superrotation is the transport of angular momentum by the mean meridional circulation, that pumps angular momentum through unbalanced surface exchanges and accelerates the atmosphere until it is balanced by eddy transport terms and the surface exchanges also balance between high and low latitudes.

\subsection{Titan}

\subsubsection{Observations of Titan's Atmospheric Circulation}

Though the first observations of Titan with the Voyager spacecrafts in the early 1980's revealed that Titan is covered with a photochemical haze, this cover displays almost no contrasts that would allow, as in the case of Venus's clouds, to track the flow and measure winds. The atmospheric circulation therefore remained unknown for a long time. From the Voyager/IRIS infrared spectra, atmospheric temperatures were obtained, and allowed to obtain very preliminary indications that high velocity zonal winds should be present in Titan's atmosphere, using the thermal wind equation (Flasar et al. 1981; Flasar and Conrath 1990). In the 1990's, the first GCM simulations of Titan's atmosphere (Del Genio et al. 1993; Hourdin et al. 1995) predicted that it should be in superrotation, with winds up to $200 \mathrm{~m} / \mathrm{s}$ in the winter stratosphere (the rotation period of Titan is close to 16 Earth days, yielding equatorial surface rotation speed slightly below $12 \mathrm{~m} / \mathrm{s}$ ). Observations to support this were obtained from Earth-based observations, with stellar occultations. The measurements of the shapes of the caustics during central flash events, which sample the atmosphere near the 0.25 -mbar level, allow to retrieve the density distribution of the atmosphere, and in particular the latitudinal profile of the zonal wind velocity in the region (Hubbard et al. 1993, Titan N. summer; Bouchez 2004, late N. autumn; Sicardy et al. 2006, early N. winter). These observations do not indicate the wind direction though.

The first direct observations were obtained using the Doppler-shift technique. Groundbased observatories offered a means of measuring the direction as well as magnitude of stratospheric winds by measuring Doppler shifts of spectral lines emitted from molecular 
Fig. 8 (a) Zonal wind distribution obtained from the thermal wind equation applied to Cassini/CIRS temperature retrievals from the period 2008-2009 (adapted from Achterberg et al. 2011). (b) Zonal wind measurements obtained from various techniques, including the Huygens descent (Bird et al. 2005) (figure from Hörst 2017)

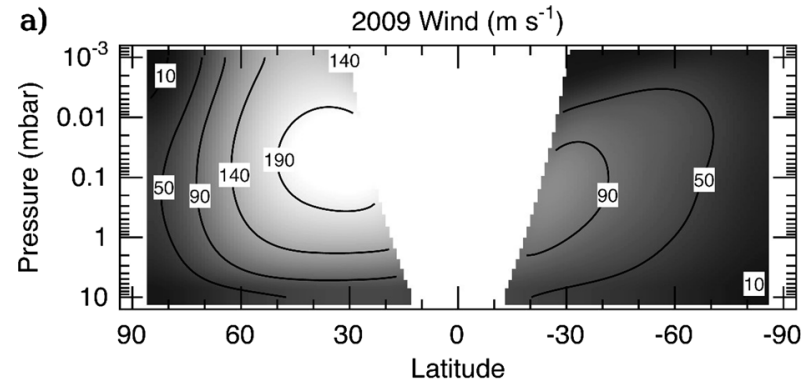

b)

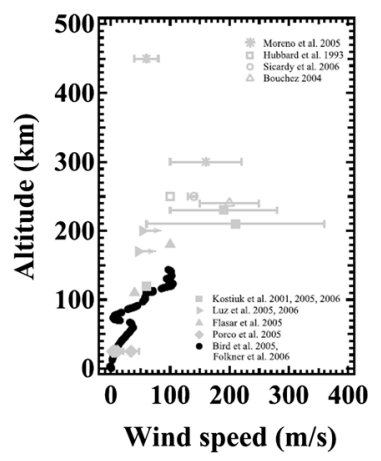

constituents of the atmosphere, albeit at limited spatial resolution. Kostiuk et al. (2001) used infrared heterodyne spectroscopy on Titan's ethane emission lines near $12 \mu \mathrm{m}$ to measure the differential Doppler shift between the eastern and western limbs of Titan, yielding a zonal wind velocity in the direction of the surface rotation (prograde) greater than $200 \mathrm{~m} / \mathrm{s}$ at pressures around $1 \mathrm{mbar}$ (altitudes around $220 \mathrm{~km}$ ). These results were later confirmed by further observations (Kostiuk et al. 2005, 2006, 2010). Luz et al. (2005, 2006) used a similar Doppler-shift technique on high-resolution visible spectrum observations to infer prograde wind speeds above $60 \mathrm{~m} / \mathrm{s}$ around $1 \mathrm{mbar}$. Millimeter-wave interferometry on rotational lines of nitriles probed higher altitudes, retrieving winds of roughly $160 \mathrm{~m} / \mathrm{s}$ near the $0.1 \mathrm{mbar}$ pressure region (altitudes near $300 \mathrm{~km}$ ) and $60 \mathrm{~m} / \mathrm{s}$ near $0.01 \mathrm{mbar}(\sim 450 \mathrm{~km}$ ) (Moreno et al. 2005).

The Cassini-Huygens mission arrived in the Saturnian system in 2004 and was a major tool to observe Titan's circulation during almost half a Titan's year. First directly, with the DWE experiment on-board Huygens, that measured the vertical profile of the zonal wind from around $140 \mathrm{~km}$ altitude (more than $100 \mathrm{~m} / \mathrm{s}$ ) down to the surface (Bird et al. 2005). Then with the thermal wind equation applied to the Cassini/CIRS temperature retrievals (Flasar et al. 2005; Achterberg et al. 2008, 2011). Taking advantage of the limb soundings done during each of Titan's flybys by this infrared spectrometer, temperature maps up to a few $\mu$ bar and at most latitudes were obtained, allowing to retrieve thermal winds, albeit in the equatorial regions. Seasonal variations of the zonal wind were obtained thanks to the length of the Cassini mission (Achterberg et al. 2011).

These measurements are illustrated in Fig. 8.

\subsubsection{A Short History of Titan's General Circulation Models}

As for Venus, efforts to develop Titan GCMs arose after the two Voyager spacecraft sent images of this dense and haze-covered atmosphere. After the study of Del Genio et al. (1993), 
that investigated the circulation of a slowly rotating atmosphere with Titan-like opacity distribution, the first Titan-dedicated GCM, developed at Laboratoire de Meteorologie Dynamique (LMD), was published by Hourdin et al. (1995). These models were able to predict superrotation in a form that was later observed. The vertical range of the LMD Titan GCM was from the surface up to $\sim 250 \mathrm{~km}$. Radiative transfer was computed using a horizontally uniform vertical profile of haze and gases (inherited from the model developed by McKay et al. 1989) everywhere on the planet. The same radiative transfer model was used by another GCM developed in Köln (Tokano et al. 1999), that failed to produced strong enough superrotation. However, the development of the Köln model continued up to now, with studies mostly dedicated to Titan's troposphere (e.g. among the most recent works, Tokano et al. 2011; Tokano 2012, 2019).

At the same time, some studies led to the idea that couplings between the circulation and the distribution of opacity sources may play a significant role in Titan's climate (e.g. Bézard et al. 1995). However, including photochemical (describing the composition) and microphysical (describing the haze) models into circulation models to study the impact of opacity sources variations on radiative transfer and the impact of atmospheric transport on their distribution was a complex task. Rannou et al. (2002, 2004) developed a two-dimensional axisymmetric (latitude-altitude) version of the LMD Titan GCM. This two-dimensional climate model (2D-CM) developed at IPSL (Institut Pierre-Simon Laplace) covered altitudes from the surface to $\sim 500 \mathrm{~km}$. Non-axisymmetric barotropic waves that could not be present in this model were parameterized based on the LMD Titan GCM results (Luz and Hourdin 2003; Luz et al. 2003). This model demonstrated the impact of the variations in the haze distribution on the dynamics, and interpreted several features observed in the haze layer. This model also included the variations of the trace gas composition (Hourdin et al. 2004; Crespin et al. 2008), which allowed to interpret the enrichments observed in trace gases in the winter polar regions. The couplings between haze, dynamics, and composition were reviewed in Lebonnois et al. (2009). The methane cycle in the troposphere was studied with this model (Rannou et al. 2006) as well as with another simple two-dimensional Titan model dedicated to the troposphere (Mitchell et al. 2006), which was later extended to a full GCM using a gray atmosphere (Mitchell et al. 2011). Schneider et al. (2012) was another similar GCM (using the FMS spectral dynamical core, Flexible Modeling System of the Geophysical Fluid Dynamics Laboratory) dedicated to the troposphere and to the study of the methane cycle.

The most recent GCMs that successfully reproduce superrotation up to the upper stratosphere are Titan-WRF (Newman et al. 2011), the IPSL Titan GCM that includes all the couplings of the IPSL Titan 2D-CM (Lebonnois et al. 2012b), and TAM (Titan Atmospheric Model, Lora et al. 2015), that uses the FMS spectral dynamical core. Both Titan-WRF and TAM include methane moist processes for a most realistic description of the tropospherical methane cycle. These models illustrated the crucial role of planetary-scale waves in the superrotation mechanism for Titan's atmosphere. Together with the latest version of the Köln Titan GCM (Tokano 2019), they have produced the highest-fidelity simulations describing Titan's climate.

\subsubsection{Current Interpretation of Titan's Superrotation}

A recent review of Titan's atmospheric dynamics was published in Lebonnois et al. (2014). Due to Saturn's inclination, Titan's circulation is affected by the seasonal cycle, similarly to the Earth. The zonal wind field modeled by the most recent and complete Titan GCMs is illustrated in Fig. 9. The whole atmosphere is in superrotation, though the dominant jet 

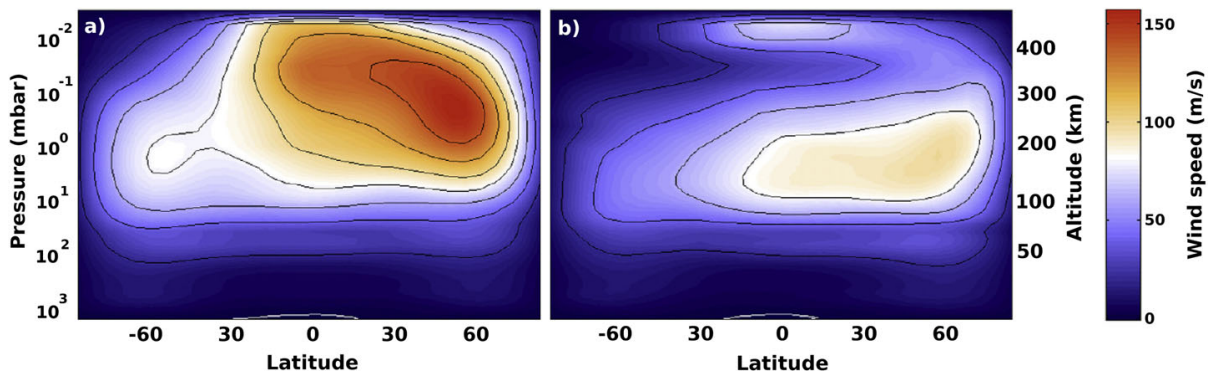

Fig. 9 Zonal-mean zonal wind fields (in m/s) modeled by the TAM Titan GCM, for (a) northern mid-fall and (b) mid-winter. An approximate altitude scale is given for reference. Adapted from Lora et al. (2015)
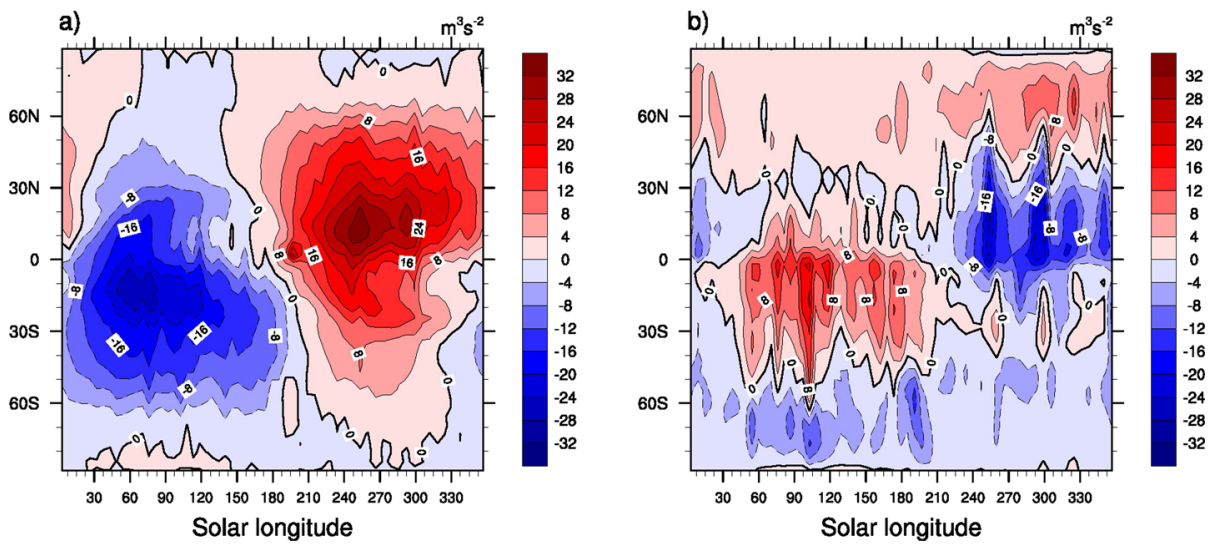

Fig. 10 Seasonal variations of the vertically-integrated latitudinal transport of angular momentum by (a) mean meridional circulation and (b) transient waves. Unit is $10^{3} \mathrm{~m}^{3} / \mathrm{s}^{2}$, positive values are northward. From Lebonnois et al. (2012b)

is located in the winter hemisphere and shifts from one hemisphere to the other during the equinox season.

The LMD Titan GCM (Hourdin et al. 1995) was the first GCM to clearly support the Gierasch-Rossow-Williams mechanism (Gierasch 1975; Rossow and Williams 1979), with a balance between angular momentum transport by the mean meridional circulation being compensated by the equatorward transport due to stratospheric barotropic planetary-scale waves. In the recent simulations of the Titan-WRF GCM (Newman et al. 2011) and of the IPSL Titan GCM (Lebonnois et al. 2012b), barotropic activity in the stratosphere during winter episodically transports angular momentum against the transport due to the mean meridional circulation, allowing equatorial superrotation to be maintained. This is illustrated in the case of the IPSL Titan GCM in Fig. 10. This figure also shows the seasonal variations of the angular momentum balance. During most of the year, the dominant meridional circulation is composed of large pole-to-pole stratospheric cell, with ascending motion in the summer hemisphere and descending motions over the winter hemisphere. This results in a summer-to-winter angular momentum transport. During a transition phase over each equinox, the ascending branch moves from one hemisphere to the other as the season changes. 


\subsection{What do We Learn when Comparing Venus and Titan?}

These two examples of superrotation present in the Solar System represent a unique opportunity to study closely this phenomenon in two different cases. Several parameters distinguish Venus from Titan, and this is of interest when wondering about the causes and robustness of superrotation in terrestrial planets.

- The rotation rates of Earth, Titan and Venus are separated by one order of magnitude between each of these planets. This is an opportunity to validate the influence of this parameter on the development of superrotation.

- The presence of a seasonal cycle on Titan illustrates that despite significant variations in the circulation during the year, the annual average situation appears similar to Venus in terms of mean meridional circulation and angular momentum budget. However, the wave activity is significantly affected by seasonal variations.

- The role of thermal tides in the atmosphere of Venus is a significant difference with the atmospheric circulation of Titan, where long radiative timescales prevent diurnal tides to affect the circulation. Their active part in the angular momentum budget shows that superrotation may be attained through different types of angular momentum transport balance.

An interesting common feature is the dominant absorption of the solar flux within the atmosphere, and not near the surface. Whether this is a crucial feature for superrotation to develop remains to be clarified.

\section{Superrotation on the Gas Giants}

\subsection{Observations of Jupiter and Saturn's Atmospheric Circulation}

Both Jupiter and Saturn are characterized by strong east-west zonal flows at their observed cloud-level. Jupiter has six such jet-streams in each hemisphere with maximum wind velocities reaching $140 \mathrm{~m} / \mathrm{s}$ at latitude $23^{\circ} \mathrm{N}$ relative to the System III spin rate (Porco et al. 2003). The equatorial region is superrotating with wind speeds exceeding $100 \mathrm{~m} / \mathrm{s}$ (Fig. 11). Saturn has a broader wind structure around the equator with superrotating winds reaching nearly latitude $30^{\circ}$ in both hemispheres, and wind velocities of over $400 \mathrm{~m} / \mathrm{s}$ (Sánchez-Lavega et al. 2000, 2003) (Fig. 11). For both planets these observations are obtained by cloud tracking of features within the cloud-level, and therefore the measurements are limited to the level of the cloud-motion, roughly between 0.1 and 1 bars (Vasavada and Showman 2005). The only direct observation of sub-cloud level winds comes from the Galileo probe that descended into the planet in 1995, and showed, at that location $\left(6^{\circ} \mathrm{N}\right)$, an increase in zonal velocity from $80 \mathrm{~m} / \mathrm{s}$ at the 1 bar level to $160 \mathrm{~m} / \mathrm{s}$ at the 4 bar level, and then a constant wind speed down to the 24 bar level where the probe was lost (Atkinson et al. 1996). However, the probe entered a "hot spot", regions occupying just $0.1-0.5 \%$ of Jupiter's surface area, but dominate its thermal emission to space at the 5- $\mu \mathrm{m}$ wavelength and are likely depleted in cloud abundance and water content and thus may not be a good representation of the general flow (Orton et al. 1998; Showman and Dowling 2000). Indirect observations of the sub-cloud level winds using cloud tracked wind velocities have been inferred by Dowling and Ingersoll (1988, 1989), who suggest based on arguments of conservation of potential vorticity (Pedlosky 1987) that there must be nonzero zonal winds underlying the Great Red Spot. Conrath et al. (1981) and Gierasch et al. (1986) used thermal-wind arguments 
Fig. 11 Zonal mean wind profile $(\mathrm{m} / \mathrm{s})$ as function of latitude for Jupiter (Porco et al. 2003) (left) and Saturn (Sánchez-Lavega 2000) (right). The angular momentum conserving zonal velocity curve (Eq. (3)) of a fluid parcel beginning at rest at the equator and moving poleward is shown in red

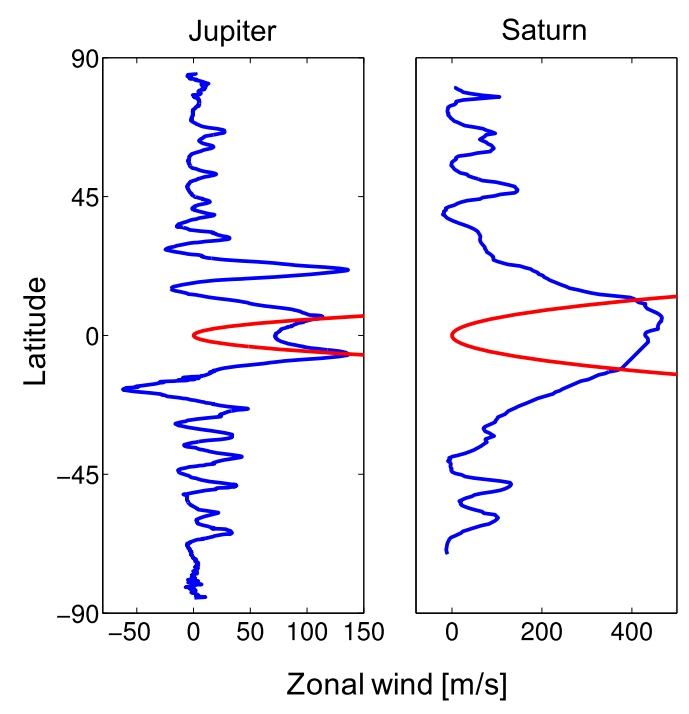

to show that deep winds are expected beneath the cloud level. However, these deductions are limited to less than the outer $1 \%$ of the radius of the planet, and therefore do not infer much about the deep winds. At the cloud-level, both planets show a strong correlation between the zonal flows and the meridional momentum flux convergence (Salyk et al. 2006; Del Genio et al. 2007), implying that at least at the cloud-level the eastward (westward) jets are strongly related to regions of momentum flux convergence (divergence).

Significant progress in revealing the sub-cloud motion on Jupiter and Saturn has been established by the recent gravitational measurements by Juno and Cassini, respectively (Iess et al. 2018, 2019). Both missions encountered the planets in close flybys enabling accurate measurements of the gravity field (Bolton et al. 2017; Edgington and Spilker 2016). The measurements are so precise that they have enabled detection of the gravity signature of the deep flows and determination of their depth and vertical profile (Kaspi et al. 2010, 2018). For the case of Jupiter, the gravity field was found to be north-south asymmetric. As there are no expected hemispherical asymmetries on a gas giant other than the observed asymmetry in the cloud-level wind structure (Fig. 11), this asymmetric gravity measurement must be a signature of deep dynamics (Kaspi 2013). Inversion of the measured gravitational field of Jupiter into the zonal flows implies that the zonal flow profile, as it appears at the cloudlevel, extends down to a depth of about $3000 \mathrm{~km}$ with a decaying zonal wind profile (Kaspi et al. 2018). The fact that the same meridional profile of the zonal flow appears at great depth has significant implications for the mechanisms driving the zonal jets. For Saturn, the odd gravity harmonics have smaller amplitudes compared the noise level of the measurements, but the even harmonics beyond $\mathrm{J}_{4}$ were found to deviate significantly from those of a uniformly rotating fluid body indicating that the depth of the flows is roughly $9000 \mathrm{~km}$ and exhibiting a meridional profile close to the observed cloud-level wind (Galanti et al. 2019). Despite the difference in the penetration depth of the winds, both for Jupiter and Saturn this depth corresponds to the radial distance at which magnetohydrodynamic theory predicts that magnetic dissipation begins to play a role in dissipating the deep flow ( $\sim 10^{5}$ bar deep). This again has significant implications for the mechanisms driving and damping these flows. 


\subsection{A Short History of Modeling Jupiter and Saturn's Zonal Flows}

There have been two general approaches for explaining the observed strong east-west zonal winds on Jupiter and Saturn. The first assumes the dynamics are shallow, such as on a terrestrial planet, and therefore the strong east-west flows can result from 2D geostrophic turbulence (Rhines 1975, 1979). Several studies have suggested mechanisms for the formation of jets in such systems from either shallow decaying turbulence (e.g., Cho and Polvani 1996a, 1996b; Scott and Polvani 2007), or forced turbulence in both barotropic (Williams 1978; Showman et al. 2006; Showman 2007; Farrell and Ioannou 2009; Chemke and Kaspi 2015) and baroclinic systems (Williams 2003; Kaspi and Flierl 2007; Lian and Showman 2008). In the last decade a number of terrestrial-type GCM studies have produced multiple jets that are consistent with the observed flows on all four giant planets including the superrotation on Jupiter and Saturn (Lian and Showman 2010; Liu and Schneider 2010). Recent work with new GCMs that include more realistic radiation schemes (Guerlet et al. 2014; Spiga et al. 2020) and extend down below the water condensation level ( $\sim 10$ bar) reaching depths $\sim 20$ bar (Young et al. 2019$)$ show consistency with observations and compelling mechanisms for superrotation. The alternative approach suggests that the observed jets are the surface manifestation of convective columns originating from the hot interiors of the giant planets (Busse 1976; Busse 1994). It has been demonstrated in 3D numerical rotating convection models, extending to depths of Mbars, that the interaction of such internal convection can lead to equatorial superrotation and multiple jets caused by convective columns (Aurnou and Olson 2001; Christensen 2001; Wicht et al. 2002; Heimpel et al. 2005, 2016; Kaspi et al. 2009; Jones and Kuzanyan 2009; Gastine et al. 2013; Chan and Mayr 2013; Heimpel et al. 2016; Dietrich and Jones 2018). An intermediate approach suggests that the jets might be triggered in the shallow weather layer by the perturbing effects of convection influencing the base of the weather layer (Showman et al. 2019). We shortly review both approaches below.

\subsubsection{Shallow Models}

Meteorology developed as a study of the thin atmospheric layer on Earth, and ideas from this field have been applied to the giant planets. A primary focus has been the appearance of zonal jets during the evolution of geostrophic turbulence (e.g., Vallis and Maltrud 1993; Panetta 1993; Cho and Polvani 1996a, 1996b; Huang and Robinson 1998; Manifori and Young 1999; Williams 2003; Smith 2004; Ishioka 2007; Scott and Polvani 2007; Kaspi and Flierl 2007; Hayashi 2007; Showman 2007; Farrell and Ioannou 2009). The first to apply such ideas to Jupiter was Gareth Williams, who used both barotropic (Williams 1978) and baroclinic (Williams 1979) models to show that an imposed turbulent eddy field can lead to an inverse energy cascade generating multiple jets on the order of the Rhines scale $\left(L_{\beta}=\left(u^{\prime} / \beta\right)^{1 / 2}\right)$. Williams (1979) and Panetta (1993) demonstrated that jets can emerge from baroclinic instability in a two-layer model with an imposed thermal gradient, allowing transfer of energy from the upper to the lower layer and results in an equivalent barotropic jet. Williams (2003) produced jets in a baroclinic spherical primitive equation model, showing that depending on details of the stratification and shear, the jets can migrate equatorward. Cho and Polvani (1996a, 1996b) showed that a shallow water layer on a sphere forced by imposed eddies evolves to a set of zonal jets at the lower latitudes. All these models assume an artificial boundary at a shallow depth (several scale heights at most), which is inconsistent with the recent Juno and Cassini gravity measurements. Also, in most cases, the jets emerging from these models are barotropically stable-in contrast to the observed 
Fig. 12 Example of multiple jets driven by shallow geostrophic turbulence in a Saturn GCM (Spiga et al. 2020). Shown is the instantaneous zonal velocity field at the 1.5 bar pressure level

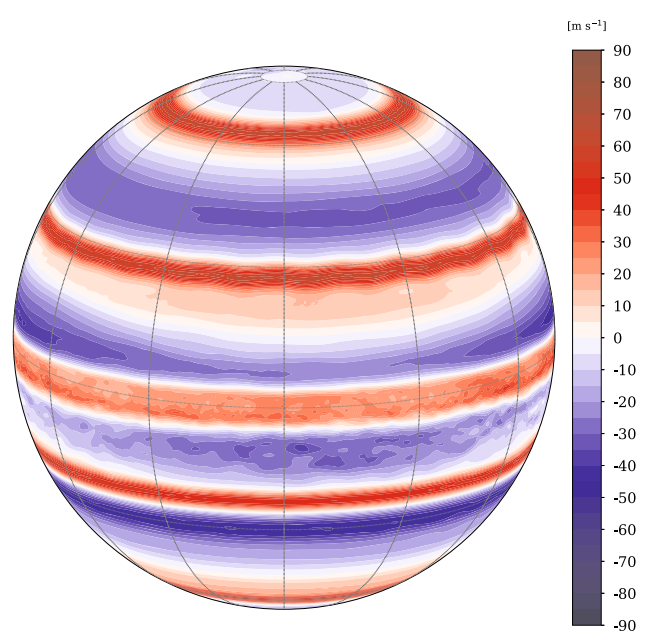

winds for which $\beta-u_{y y}<0$ at some latitudes. Most models of this type produce an equatorial jet that flows westward rather than eastward as on Jupiter and Saturn, since without any particular forcing at the equator, Hide's theorem implies that there can be no angular momentum maxima at the equator. One exception is the model of Scott and Polvani (2008), which develops equatorial superrotation in a shallow-water model when large-scale energy dissipation by radiative relaxation is taken into account.

More recently, there has been significant progress in understanding this problem using 3D atmospheric idealized GCMs. Three-dimensional models can explicitly resolve the turbulent energy generation, and therefore do not need to inject the turbulence artificially as in the models discussed above. These models likewise exhibit multiple zonal jets via the Rhines effect, spontaneously producing an eastward equatorial jet as on Jupiter and Saturn (Fig. 12). Lian and Showman (2010) suggest that latent heating associated with condensation of water vapor can drive the superrotation. They performed the first models of jet formation that include an active hydrological cycle for water, which has long been considered to play an important role in the dynamics (e.g., Barcilon and Gierasch 1970; Ingersoll et al. 2000). Lian and Showman (2010) find that the abundance of water vapor, which is higher on Uranus and Neptune, is the key factor controlling the direction of the equatorial flow, although the planetary rotation rate and radius also exert an influence on the equatorial jet direction and speed. Alternately, using bottom drag to parametrize interior ohmic dissipation, Liu and Schneider (2010) showed that whether the equatorial jet is superrotating or subrotating depends on if the intrinsic heat fluxes are strong enough to generate equatorial Rossby waves, which cause an equatorial eddy momentum flux convergence, leading to superrotation. Similarly, Young et al. (2019) find that whether their model develops superrotation or not depends on the level of internal heating. In their model when there is internal heating the jets migrate equatorward creating a flux convergence of angular momentum and thus superrotation.

Showman et al. (2019) showed that convective perturbations at the bottom of the stratified weather layer can induce multiple zonal jets, as well as a stratospheric oscillation analogous to the quasi-biennial oscillation (QBO) on Earth, comprising vertically stacked eastward (superrotating) and westward (subrotating) equatorial jets that migrate downward over time. Such stratospheric oscillations have been observed on Jupiter with a period close to 4 years, called the quasi-quadrennial oscillation (QQO) and on Saturn with a period of 15 years (half 
a Saturn year), and often termed the Saturn Semi-annual oscillation. Analogous to the dynamics of Earth's QBO, the downward migration of these vertically stacked superrotating and subrotating stratospheric jets in Showman et al. (2019)'s models are driven by the vertical propagation of equatorial waves that are absorbed at critical levels in the equatorial stratosphere.

\subsubsection{Deep Models}

A different approach for explaining the zonal jets on the giant planets comes from interior convection models. Following on a series of seminal papers by F. H. Busse in the 1970s, under the assumption that interior convection homogenizes entropy gradients such that the interior may be nearly barotropic, resulting in the formation of Taylor columns in the interior, he proposed that the nonlinear interaction of these convectively driven columns can generate equatorial superrotation and possibly high latitude east-west jet streams (Busse 1970, 1976, 1983, 1994). Given the recent gravitational measurements (Sect. 3.1) it is clear that such structures penetrating throughout the planet cannot exist, yet given the depth of the flow and its likely alignment with the axis of rotation (Kaspi et al. 2018), the mechanisms for superrotation provided by this approach are still applicable. Those are discussed here.

Theories of jet formation and superrotation due to interior convection are based on the idea that convectively generated Reynolds stresses in the molecular envelopes of the giant planets can cause large-scale, organized differential rotation within the interior, which manifests at the atmospheric level as multiple zonal jets. Within a rapidly rotating planet, such as the gas giants of the solar system, as long as the Rossby number is small, surfaces of constant angular momentum are aligned with the axis of rotation. Conservation of angular momentum then implies that to leading order $\mathbf{u} \cdot \nabla \mathrm{M}=0$, where $\mathbf{u}$ is the $3 \mathrm{D}$ flow velocity and $\mathrm{M}$ is angular momentum per unit mass, meaning that the meridional circulation will be aligned with the direction of the spin axis (Kaspi et al. 2009; Schneider and Liu 2009). Therefore, the convectively driven flow is not only driven by buoyancy (in which case it would be mainly in the radial direction), but also by the limitation that the flow must be aligned with the direction of the axis of rotation (Kaspi et al. 2009; O'Neill and Kaspi 2016). In the simple barotropic and incompressible limit this leads to the Taylor-Proudman effect (Taylor 1923; Pedlosky 1987), meaning $\boldsymbol{\Omega} \cdot \nabla \mathbf{u}=0$ (where $\boldsymbol{\Omega}$ is the rotation vector), so that the flow velocity remains constant along the direction of the axis of rotation. Even for the compressible case, the zonal component of this expression remains valid meaning that the zonal velocity should be constant along the direction of the spin axis (Showman and Kaspi 2013). However, numerical models show that the convective motion will still be aligned with the axis of rotation also in compressible and baroclinic cases (Kaspi et al. 2009; Jones and Kuzanyan 2009), yet the zonal velocity can still change along this direction. Figure 13 (left) shows a schematic of such convection columns as illustrated by Busse (2002).

Generation of superrotation by internal convection columns is due to the fact that these convection columns are not only aligned parallel to the axis of rotation, but are also noncircular in cross section, but rather elongated with an axis that tilts with radius, in direction of the rotation, such that angular momentum is transported radially (Fig. 13, middle). The tilt results from the natural solution of the most unstable modes on the sphere, and has been shown both analytically by linear stability analysis (Zhang and Busse 1987) and numerically in Zhang (1992). The direction of the tilt is set by the boundary conditions and has been shown to depend on whether the outer boundary is convex or concave, and within the sphere are tilted in the direction of the rotation (meaning that the tilt is eastward as one moves away from the rotation axis). The tilted columns cause the velocity anomaly along the columns, 

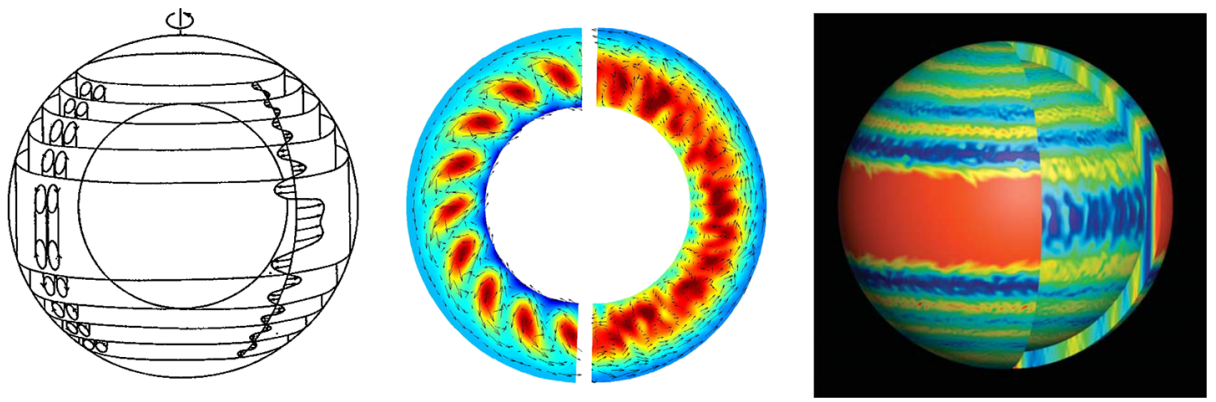

Fig. 13 Superrotation in deep models: left: Busse's picture of columnar convection generating east-west flows (Busse 1994). Middle: The equatorial plane vorticity (color), velocity (arrows) for cases of critical (left) and supercritical (right) Rayleigh number simulations in a deep anelastic GCM (Showman et al. 2011). These tilted columns result in an outward momentum flux, which at the equator converge to give superrotation. Right: The zonal velocity field including the equatorial superrotation (Heimpel et al. 2005)

in the azimuthal direction $u^{\prime}$, and in the direction pointing outward from the spin axis $\left(v_{\perp}^{\prime}\right)$, to be always positively correlated so that the eddy fluxes (Reynolds stresses) $u^{\prime} v_{\perp}^{\prime}>0$ (see arrows in Fig. 13, middle). This effect results in a net eddy angular momentum flux outward from the convection columns, and thus an eddy momentum flux convergence in the equatorial region outside of the convection columns. In essence, this mechanism is similar to the shallow one (Sect. 3.2), where meridional flux convergence of eastward momentum leads to eastward jets, only here the momentum flux convergence is in both the meridional and vertical direction leading to convergence of eastward momentum at the equator (due to the sphericity of the planet) in the outer regions, and concomitantly a divergence of eastward momentum inward of the convection columns. This angular momentum transfer induces an eastward superrotating mean flow outside of the convection columns and a westward mean flow inward of the convection columns (Fig. 13, right). The direction of the mean flow supports the direction of the tilt in the convection columns and therefore a positive feedback between the tilted columns and the mean flow ensures the stability of the columns (see arrows in Fig. 13, middle). Simulations with oppositely rotating columns show that although they emerge for weakly convective systems they quickly get sheared away by the mean flow. Despite the appeal of the 3D character of these models their main flaw is that they must be strongly over forced (Showman et al. 2011) in order to numerically converge on reasonable time scales, and thus are a good qualitative tool, but not a quantitative one.

\subsection{What do We Learn when Comparing Jupiter and Saturn?}

The similarities and differences between the two gas giants, combined with recent new data from Juno and Cassini, provide an opportunity to compare the two planets with the strongest superrotation in the Solar System.

- The recent gravity measurements from Juno and Cassini imply that the depth of the flow on Saturn is likely three times deeper than on Jupiter (Kaspi et al. 2018; Galanti et al. 2019). Yet, the mean density on Saturn is three times lower, implying that in both cases the flows extend down to nearly $10^{5}$ bars ( $3000 \mathrm{~km}$ on Jupiter and $9000 \mathrm{~km}$ on Saturn). This is also the depth where the flow becomes semi-conducting, meaning that the magnetic field might be interacting with the zonal flows at depth (Kaspi et al. 2020), implying that the termination of the jets at these depths may be due to Ohmic dissipation (Liu et al. 2008; Cao and Stevenson 2017). 
- The latitudinal width of the equatorial eastward flow on both planets is very different, extending to about latitude $13^{\circ}$ on Jupiter and $31^{\circ}$ on Saturn (Fig. 11). However, this latitudinal extent is consistent with considering the depth implied by the gravity measurements, and extending it outward along the direction of the spin axis until it outcrops the surface (Fig. 13). Thus, the wider superrotation on Saturn is consistent with the Saturnian flows being deeper, implying that the interaction with the magnetic field (above) provides a possible explanation to the wider extent of the superrotation on Saturn (Kaspi et al. 2020).

- Although no single mechanism for superrotation has been identified, it is likely that it is driven by an eddy momentum flux convergence at the equator that can be mostly a meridional convergence (shallow mechanisms) or a 2D meridional-vertical convergence (deep mechanisms). The bigger uncertainty is the source of the eddies, which can be convective, or due to baroclinic/barotropic instability. Further constraints may be obtained by better constraining the magnetic field anomalies, and how these interact with the flow

(Galanti et al. 2017; Connerney et al. 2018; Duer et al. 2019; Moore et al. 2019).

\section{Superrotation on Exoplanets}

\subsection{Hot Jupiters}

The most easily characterizable exoplanets are transiting exoplanets that orbit very close to their parent stars; therefore, the majority of observational, theoretical, and modeling work has been done for these strongly irradiated planets rather than for exoplanets more distant from their stars. Close-in planets are subject to tidal forces that cause them to become tidally despun; therefore, while observational constraints on rotation rate are currently scarce, most close-in exoplanets are assumed to be synchronously rotating, with permanent daysides and nightsides. For example, the canonical hot Jupiter - a giant planet at orbital distances $\sim 0.05$ AU from a Sun-like star-exhibits a spindown time of order $10^{6}$ years, far shorter than the multi-billion year age of typical systems (Guillot et al. 1996). Early GCM simulations of such planets preceded observations and adopted highly idealized forcing schemes and predicted that the strong day-night thermal forcing would lead to the presence of a strong superrotating equatorial jet with zonal-mean wind speeds of several $\mathrm{km} / \mathrm{s}$ (Showman and Guillot 2002; Cooper and Showman 2005). On a Jupiter-sized planet, such a wind speed implies a characteristic timescale for air to advect across a hemisphere of order $10^{5} \mathrm{~s}$. Interestingly, the high temperature of hot Jupiters - commonly $1000 \mathrm{~K}$ or more-also implies rather short radiative timescales of order $10^{5} \mathrm{~s}$ (significantly shorter than the radiative timescale in Earth's atmosphere, for example). The approximate matchup in the zonal advection and radiative timescales implies that the superrotating jet causes an eastward longitudinal displacement of the dayside hot region. This effect was first predicted by Showman and Guillot (2002), who suggested that it would be observable in thermal phase curves of hot Jupiters. Subsequent observations from the Spitzer Space Telescope first confirmed this prediction for the hot Jupiter HD 189733b (Knutson et al. 2007), and later IR observations from the Spitzer and Hubble Space Telescopes have detected such an eastward offset of the hot spot on the majority of hot Jupiters that have been observed (see Heng and Showman 2015 for a review). Thus, the indirect observational inference is that many if not most of these planets exhibit equatorial superrotation in their atmospheres. Moreover, the Doppler signature of atmospheric winds has been observationally determined for one hot Jupiter, HD 189733b (Louden and Wheatley 2015); the observations separately isolated the Doppler signature on 
the leading and trailing limbs and showed that the atmosphere exhibits winds that appear to be superrotating at speeds of several $\mathrm{km} / \mathrm{s}$.

Note that beyond the early studies quoted above, many additional GCM codes have been employed to investigate the problem of hot Jupiter atmospheric circulation over the past ten years; interestingly, most of these models agree reasonably well in their qualitative predictions, including the existence of equatorial superrotation in most cases (e.g., Menou and Rauscher 2009; Rauscher and Menou 2010, 2012, 2013; Dobbs-Dixon and Lin 2008; Dobbs-Dixon and Agol 2013; Heng et al. 2011a, 2011b; Menou 2012; Mayne et al. 2014, 2017; Showman et al. 2009, 2015; Parmentier et al. 2013, 2016; Kataria et al. 2015, 2016; Lewis et al. 2010, 2013; Cho et al. 2015; Zhang and Showman 2017; Komacek et al. 2017; Roman and Rauscher 2017; Mendonca et al. 2018; and others). Interestingly, the presence and qualitative properties of the superrotation seem to be robust to model assumptions, numerics, and the detailed formulation of the day-night thermal forcing (e.g., Showman and Polvani 2011). This provides an interesting contrast to Venus, where model results seem quite sensitive to numerics, and even Jupiter and Saturn, for which numerical circulation models can easily exhibit either eastward or westward equatorial flow depending on detailed assumptions (e.g., see Vasavada and Showman 2005 for a review). This fact suggests that the mechanism driving superrotation on hot Jupiters is extremely robust.

\subsection{Tidally-Locked Terrestrial Exoplanets}

Atmospheric superrotation on terrestrial exoplanets has likewise long been predicted. Because of interest in habitability, terrestrial exoplanet GCMs have emphasized planets in the habitable zones of their stars, that is, in temperature regimes similar to that of Earth. The most observable of this class of habitable-zone exoplanet are terrestrial exoplanets orbiting M dwarfs, which are cool red dwarf stars. Because such stars are dim (that is, their luminosity is much less than that of the Sun), habitable-zone temperatures only occur when planets orbiting such stars are extremely close-in, with orbital periods typically of order 10-30 days depending on the star. These distances are close enough that, like the hot Jupiters, such planets are expected to become tidally despun into a synchronously rotating state, with permanent daysides and nightsides. Thus, these planets likely exhibit rotation periods of order 10-30 Earth days. This gives them strong dynamical similarities to Titan and Venusthey are in a slowly rotating regime where the equatorial deformation radius becomes close to a planetary radius, such that, dynamically, they are essentially "all tropics" worlds (see Showman et al. 2013a, 2013b for a review). GCMs of this class of planet began with the pioneering study of Joshi et al. (1997) and has continued with a wide range of subsequent investigations (e.g., Merlis and Schneider 2010; Edson et al. 2011; Heng and Vogt 2011; Leconte et al. 2013; Hu and Yang 2014, Yang et al. 2013, 2014, 2019 and others), many of which show that the circulations of typical tidally locked terrestrial exoplanets are expected to superrotate.

\subsection{Mechanism for Superrotation}

The dynamical mechanism for the equatorial superrotation on tidally locked exoplanets was first investigated by Showman and Polvani (2011), who showed that the day-night thermal forcing pattern leads to a standing wave pattern comprising global-scale Kelvin and Rossby waves that essentially form a Matsuno-Gill pattern on the global scale. In this pattern, the Kelvin wave component is displaced toward the east while the Rossby wave component is 
displaced toward the west (a natural outcome of the waves' group-propagation tendencies; see Gill 1980). Showman and Polvani $(2010,2011)$ showed that this pattern leads to eddyvelocity phase tilts that cause eddy angular momentum to converge onto the equator, causing equatorial superrotation. They showed that the mechanism is robust in the sense that it occurs over a wide range of forcing and damping parameters, helping to explain the ubiquity of eastward offsets in phase-curve observations of hot Jupiters, as well as the fact that superrotation occurs in a wide range of GCMs (despite differing model formulations). Showman and Polvani (2011) demonstrated the mechanism in analytical and numerical linear and nonlinear shallow-water models and in full 3D numerical simulations of the circulation on hot Jupiters. Tsai et al. (2014) extended the analytical wave formulation to 3D conditions for hot Jupiters under more specific assumptions about the damping properties (specifically that the thermal and frictional damping timescales are equal, an assumption not made by Showman and Polvani 2011). They showed that same basic wave-mean-flow interaction occurs in a $3 \mathrm{D}$ setting when the $3 \mathrm{D}$ nature of the wave structure is accounted for, thus providing additional confidence that the mechanism should indeed occur in 3D atmospheres. A variety of additional questions remain about the dynamics controlling the detailed structure of the superrotation, and how this structure depends on parameters including the strength of the radiative forcing, planetary rotation rate, and so on. As yet, there is no predictive theory for the equatorial jet speed, which is an area for future work. How the superrotation is affected by perturbations to the thermal forcing due to clouds (Roman and Rauscher 2017; Parmentier et al. 2016), magnetic effects (e.g., Rogers and Showman 2014; Rogers and Komacek 2014; Rogers 2017), and interior convection remain open questions for future research. Additional puzzles will also likely emerge as models confront better and better observations in the era of the James Webb Space Telescope.

\section{Key Observations}

Studies on the mechanism for superrotation conducted so far have reproduced, at least partly, the observed mean dynamical states with GCMs or simple dynamical models as described in the previous sections. On the other hand, observational evidence for momentum transport by non-axisymmetric eddies and the mean circulation sustaining superrotation has hardly been obtained. Among the planets having superrotating atmospheres, currently Venus and Titan are the only planets where we have chances to directly observe processes that induce angular momentum fluxes (e.g., Limaye et al. 1988a; Horinouchi et al. 2020). Rich information about atmospheric dynamics has been provided by Venus orbiters such as NASA's Pioneer Venus (Schubert et al. 1980), Soviet Venera 15 (Zasova et al. 2007), ESA's Venus Express (Svedhem et al. 2007) and JAXA's Akatsuki (Nakamura et al. 2016) as well as by groundbased telescopes (e.g., Machado et al. 2014; Peralta et al. 2017b). A wealth of information about Titan's atmospheric dynamics has been obtained by Cassini spacecraft and groundbased telescopes (e.g., Teanby et al. 2019).

Various types of eddies have been detected so far on Venus: planetary-scale traveling waves that are thought to be Rossby waves or Kelvin waves (Del Genio and Rossow 1990; Kouyama et al. 2015; Kashimura et al. 2019; Imai et al. 2019), thermal tides (Schofield and Taylor 1983; Limaye et al. 1988b; Limaye 2007; Zasova et al. 2007; Peralta et al. 2012; Ando et al. 2018; Kouyama et al. 2019), and gravity waves (Peralta et al. 2008; Tellmann et al. 2012; Piccialli et al. 2014; Ando et al. 2015; Imamura et al. 2018). The phase variation with height of the thermal tides above the clouds (Schofield and Taylor 1983) suggests 
Fig. 14 Contour plot of the latitudinal gradient of the vorticity of the mean zonal velocity deduced from Venus Express radio occultation temperatures. Units are $10^{-6} \mathrm{~s}^{-1}$. Negative regions are represented by grey. The zonal velocity $\left(\mathrm{m} \mathrm{s}^{-1}\right)$ is overplotted. (Piccialli et al. 2012)

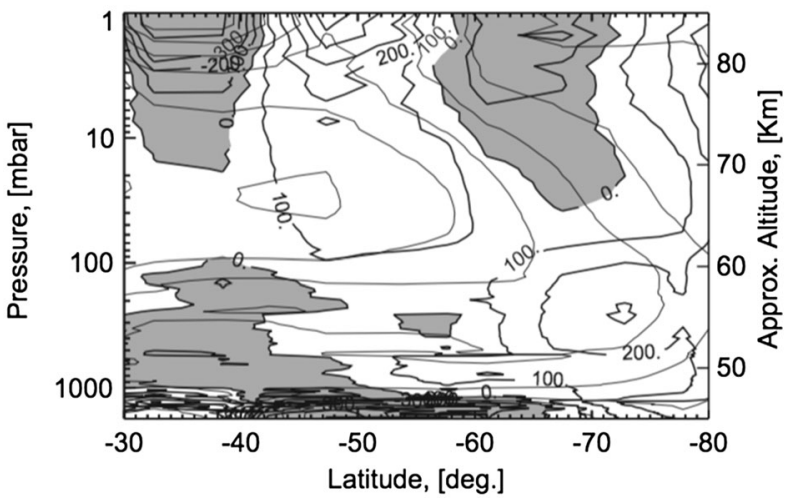

downward angular momentum transport, which can contribute to the decay of the superrotation with height above the clouds (Schubert et al. 1980). Tides that propagate downward below the clouds are potentially important for the maintenance of the superrotation (Takagi and Matsuda 2006, 2007), but the wave structures below the clouds are unknown. The observed zonal-mean structure of the cloud-level atmosphere satisfies the necessary condition for barotropic instability, i.e. the meridional gradient of the absolute vorticity changes sign within the domain (Piccialli et al. 2012) (Fig. 14), indicating a possibility of generation of eddies that induce equatorward momentum fluxes. It was also suggested based on cloud tracking that equatorward momentum transport can occur at the cloud top due to thermal tides (Horinouchi et al. 2020). An equatorial jet found in the lower cloud region by near-infrared imaging (Horinouchi et al. 2017) might imply convergence of eddy momentum fluxes. Though meridional circulation is also a crucial factor in the maintenance of the superrotation (Sect. 2.1), its structure is not well constrained by observations except that a poleward circulation is suggested around the cloud top and above from cloud tracking (Rossow et al. 1990; Hueso et al. 2015), the thermal structure (Imamura 1997; Lee et al. 2015) and the cloud structure (Imamura and Hashimoto 1998). Gravity waves and the turbulent diffusion induced by the waves are potentially important in the downgradient angular momentum transport that balances the acceleration of the superrotation; thin neutral (turbulent) layers that might have been generated by gravity waves were detected by radio occultation with a new analysis method (Imamura et al. 2018).

For understanding the angular momentum budget of the coupled atmosphere-solid planet system, the wind field near the surface, which determines the angular momentum exchange between the atmosphere and the solid planet, needs to be known. On Earth, westward winds prevail in the low latitudes and eastward winds prevail in the high latitudes near the surface, leading to an angular momentum budget that the atmosphere gets (gives) angular momentum from (to) the solid planet in the low (high) latitudes (Holton and Hakim 2013). The Earth's westward wind in the low latitudes is created by the Coriolis acceleration acting on the equatorward branch of the Hadley circulation. A qualitatively similar situation is expected for Venus, where solar radiation reaching the surface should induce a Hadley circulation (Schubert et al. 1980); equatorward trends of wind-related features seen in radar maps suggest the existence of Hadley circulation (Greeley et al. 1995). Such a circulation should induce anti-superrotational winds in the low latitudes, enabling the solid planet to pass angular momentum to the atmosphere. On the other hand, detection of mountain waves above the mountainous regions in the low latitudes by balloons (Blamont et al. 1986) and cloud imagery (Fukuhara et al. 2017; Kouyama et al. 2017; 


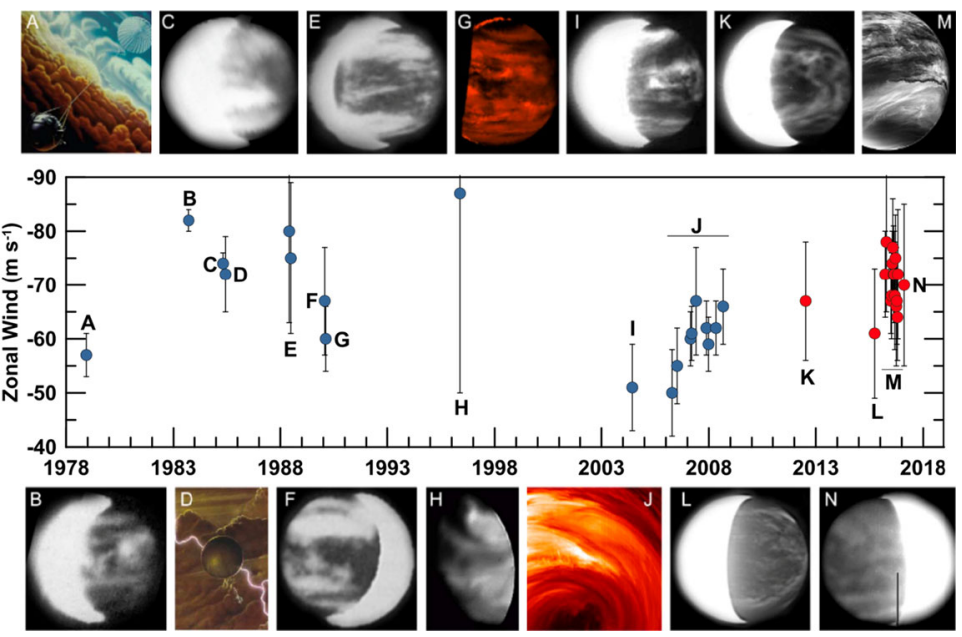

Fig. 15 Decadal variation of the zonal velocity in the low latitude $\left(<30^{\circ}\right)$ at the lower-cloud level of Venus revealed by compiling cloud tracking and in-situ measurements. The error bars stand for the standard deviation of the time averages. (Peralta et al. 2018)

Kitahara et al. 2019) implies superrotational winds blowing throughout the altitude region from the near-surface layer to the cloud top, because gravity waves cannot pass through critical levels where the horizontal phase velocity equals the background wind velocity. Stationary features indicative of mountain waves were observed also in the high-latitude cloud tops (Peralta et al. 2017a). If superrotation prevails everywhere near the surface, the atmosphere would lose excess angular momentum with time and cannot maintain the superrotation. The geographical distribution of the surface wind and its variability need to be revealed.

In addition to Venus, observations of Titan can also be used to derive some information on superrotation processes. In the troposphere, methane clouds sporadically form and evolve in a transient nature. Some of these storms have quite distinct morphologies (Turtle et al. 2011) and propagation characteristics (Schaller et al. 2009), allowing them to be identified as Kelvin and Rossby waves (Mitchell et al. 2011; Schaller et al. 2009). Although this does not prove these waves influence superrotation, their existence in the troposphere is consistent with the wave origins of the required momentum transport. Additionally, the CIRS instrument on-board Cassini enabled the evolution of the meridional distributions of temperature and of trace components to be studied. These maps can be used to infer the mean meridional circulation, as well as the latitudinal extent of the winter polar vortex (Teanby et al. 2012, 2017, 2019; Vinatier et al. 2015). These observations have shown that the mean meridional circulation is dominated by a pole-to-pole thermally-direct cell in the stratosphere for most of the year, with subsidence in the winter hemisphere and in particular in the winter polar vortex. The seasonal variations show how the ascending branch moves from one hemisphere to the other, and how the polar regions evolve as they are exiting or entering winter season. Such observations support the circulation simulated by GCMs, while providing strong constraints to improve the models.

Spatially-resolved, long-term continuous monitoring of atmospheric variables is crucial for elucidating the mechanism for superrotation. Images taken from high altitudes using wide-angle optics like those onboard Venus Express and Akatsuki are suitable for such studies (Svedhem et al. 2007; Nakamura et al. 2016). The relationship between the longterm variation of the superrotation (Fig. 15) and the variation of wave activity might give 
clues to the wave-mean flow interaction (Khatuntsev et al. 2013; Kouyama et al. 2015; Peralta et al. 2018). However, information on the dynamics obtained by the orbiters is largely limited to regions in and above the clouds, and thus key processes below the clouds will be left unsolved. Promising approaches might be, for example, long-lifetime low-altitude balloons to observe motions of the lower atmosphere, and three-dimensional tomography of the atmospheric structure using satellite-to-satellite radio occultation. Gravity measurements at higher spatial and temporal resolution than Juno using multiple spacecraft should be helpful for gas giants. To make the best use of observational data and reveal the dynamics behind them, data assimilation using GCMs (Sugimoto et al. 2017, 2019b) is promising. Observations of the atmospheres of exoplanets and evaluation of their zonal winds would enable an extensive parameter study that cannot be done with the solar system planets only.

\section{Concluding Remarks}

As described in the previous sections, superrotation is ubiquitous among planetary atmospheres, and the planetary atmospheres of the solar system show this phenomenon occurs over a wide range of planetary characteristics including fast and slow planetary rotation. Underlying common physical processes are extensively studied using numerical models, and observations of detailed atmospheric structures including eddy motions are ongoing and/or planned to constrain the mechanisms for superrotation.

The key non-axisymmetric eddies that induce upgradient angular momentum transport to maintain superrotation (Sect. 1.2) seem to be different among the planets (Table 2). Planetwide superrotation seems to occur mostly on slow rotators, where the large Rossby number allows planetary-scale waves to behave differently from the waves on fast rotators like the Earth. For example, in the atmospheres of Venus and Titan, planetary-scale waves will be generated through barotropic instability of the mid/high-latitude jets (Sect. 2) and induce equatorward fluxes of angular momentum. Coupled Kelvin-Rossby wave instability might also induce equatorward angular momentum transport according to the numerical experiments of slowly-rotating terrestrial planets (Sect. 1.3). Strong day-night contrast of the thermal forcing, which results from the solar heating of high-altitude clouds, strong irradiation from the central star, or the tidal locking of the planet, can also induce superrotation. For example, the thermal tides forced by the solar heating of Venus's high-altitude clouds can induce equatorial acceleration through the vertical transport of angular momentum (Sect. 2). Tidally-locked expoplanets, including hot Jupiters and terrestrial exoplanets orbiting $\mathrm{M}$ dwarfs, are also expected to have superrotating atmospheres; numerical experiments suggest that a thermally-forced, standing (solar-locked) wave pattern comprising Kelvin and Rossby waves can induce equatorward angular momentum transport (Sect. 4). On the other hand, the equatorial superrotation of Jupiter and Saturn seems to require fast planetary rotation. The proposed mechanisms for the acceleration, such as inverse energy cascade, baroclinic instability, equatorial Rossby waves, and the tilt of convective columns aligned parallel to the rotation axis (Sect. 3), require small Rossby numbers. Comparative studies of these different types of the wave-mean flow interaction would enable a more general understanding of superrotation.

Elucidation of circulation regimes including superrotation is also crucial to reconstructions of past climates and predictions of future climate changes. For example, it has been known that simplified Earth GCMs with enhanced tropical heating anomalies and without obliquity tend to produce a climate exhibiting equatorial superrotation (Nasuno 2008). The superrotation is driven by horizontal eddy momentum fluxes associated with 
Table 2 Classification of the planets according to angular momentum-transporting eddies

\begin{tabular}{lll}
\hline Rotation type & $\begin{array}{l}\text { Traveling waves generated } \\
\text { by instabilities }\end{array}$ & Solar-locked waves \\
\hline Slow rotator & Venus & Venus \\
& Titan & Hot Jupiters \\
& & $\begin{array}{l}\text { Tidally-locked terrestrial } \\
\text { exoplanets }\end{array}$ \\
Fast rotator & Jupiter & \\
& Saturn & \\
\hline
\end{tabular}

planetary-scale waves generated by tropical heating. In the real Earth's atmosphere, hemispheric asymmetry due to obliquity and topography seems to conceal the potential superrotation: the easterly Coriolis torque experienced by the cross-equatorial meridional circulation is responsible for maintaining the observed subrotation in the tropical upper troposphere against the westerly torque associated with tropical wave sources (Lee 1999; Kraucunas and Hartmann 2005). This implies that the Earth's atmosphere may have superrotated in the past, such as in a warmer climate where tropical wave sources were stronger. It is also suggested that the zonal wind distribution of an Earth-like exoplanet can be radically different from that of the Earth depending on the climate condition and the hemispheric asymmetry.

Ancient Venus may also have had a dynamical regime different from the current one depending on the thermal inertia of the atmosphere (Sect. 1.3). Way et al. (2016) suggested, using a GCM for ancient Venus with an atmospheric mass similar to the Earth's, that a liquid water ocean and habitable surface temperatures could have possibly existed. They assumed that the carbon cycle hides a large amount of $\mathrm{CO}_{2}$ in the crust in the presence of liquid water and continents (Walker et al. 1981). The cool climate is maintained by reflective, thick $\mathrm{H}_{2} \mathrm{O}$ clouds on the dayside, which are generated by a strong SS-AS circulation involving rising motion on the dayside (Yang et al. 2014). This result relies on the prediction that SS-AS circulation dominates over superrotation even on Venus when the atmosphere is not as thick as the present epoch.

The sciences of solar system planets and exoplanets are now going to be integrated into a truly general planetary science in many respects. Superrotation is one of the striking examples: it can play crucial roles in distributing heat and chemical species both in the solar system planets and exoplanets, thereby influencing, for example, the habitability. The study of detailed dynamical processes in the atmospheres of the solar system planets and the understanding of the diversity of exoplanet atmospheres should be combined to develop a unified atmospheric dynamics that is applicable to wide parameter ranges.

Acknowledgements We appreciate the two anonymous reviewers for their careful reading of the manuscript and providing valuable comments. We also acknowledge inspiring discussions in the ISSI Workshop "Understanding the Diversity of Planetary Atmospheres" (Bern, 2018).

Publisher's Note Springer Nature remains neutral with regard to jurisdictional claims in published maps and institutional affiliations.

Open Access This article is licensed under a Creative Commons Attribution 4.0 International License, which permits use, sharing, adaptation, distribution and reproduction in any medium or format, as long as you give appropriate credit to the original author(s) and the source, provide a link to the Creative Commons 
licence, and indicate if changes were made. The images or other third party material in this article are included in the article's Creative Commons licence, unless indicated otherwise in a credit line to the material. If material is not included in the article's Creative Commons licence and your intended use is not permitted by statutory regulation or exceeds the permitted use, you will need to obtain permission directly from the copyright holder. To view a copy of this licence, visit http://creativecommons.org/licenses/by/4.0/.

\section{References}

R.K. Achterberg, B.J. Conrath, P.J. Gierasch, F.M. Flasar, C.A. Nixon, Titan's middle-atmospheric temperatures and dynamics observed by the Cassini Composite Infrared Spectrometer. Icarus 194, 263-277 (2008). https://doi.org/10.1016/j.icarus.2007.09.029

R.K. Achterberg, P.J. Gierasch, B.J. Conrath, F.M. Flasar, C.A. Nixon, Temporal variations of Titan's middle atmospheric temperatures from 2004 to 2009 observed by Cassini/CIRS. Icarus 211, 686-698 (2011). https://doi.org/10.1016/j.icarus.2010.08.009

H. Ando, T. Imamura, T. Tsuda, S. Tellmann, M. Pätzold, B. Häusler, Vertical wavenumber spectra of gravity waves in the Venus atmosphere obtained from Venus Express radio occultation data: evidence for saturation. J. Atmos. Sci. 72, 2318-2329 (2015)

H. Ando et al., Local time dependence of the thermal structure in the Venusian equatorial upper atmosphere: comparison of Akatsuki radio occultation measurements and GCM results. J. Geophys. Res. 123, 22702280 (2018). https://doi.org/10.1029/2018JE005640

D.G. Andrews, J.R. Holton, C.B. Leovy, Middle Atmosphere Dynamics (Academic Press, New York, 1987). 489 pp.

D.H. Atkinson, J.B. Pollack, A. Seiff, Galileo Doppler measurements of the deep zonal winds at Jupiter. Science 272, 842-843 (1996)

J.M. Aurnou, P.L. Olson, Strong zonal winds from thermal convection in a rotating spherical shell. Geophys. Res. Lett. 28, 2557-2559 (2001)

M.P. Baldwin, L.J. Gray, T.J. Dunkerton, K. Hamilton, P.H. Haynes, W.J. Randel, J.R. Holton, M.J. Alexander, I. Hirota, T. Horinouchi, D.B.A. Jones, J.S. Kinnersley, C. Marquardt, K. Sato, M. Takahashi, The quasi-biennial oscillation. Rev. Geophys. 39, 179-229 (2001)

A. Barcilon, P. Gierasch, A moist, Hadley cell model for Jupiter's cloud bands. J. Atmos. Sci. 27, 550-560 (1970)

J.-L. Bertaux, I.V. Khatuntsev, A. Hauchecorne, W.J. Markiewicz, E. Marcq, S. Lebonnois, M. Patsaeva, A. Turin, A. Fedorova, Influence of Venus topography on the zonal wind and UV albedo at cloud top level: the role of stationary gravity waves. J. Geophys. Res., Planets 121, 1087-1101 (2016). https://doi.org/ 10.1002/2015JE004958

B. Bézard, A. Coustenis, C.P. McKay, Titan's stratospheric temperature asymmetry: a radiative origin? Icarus 113, 267-276 (1995)

M.K. Bird, M. Allison, S.W. Asmar, D.H. Atkinson, I.M. Avruch, R. Dutta-Roy, Y. Dzierma, P. Edenhofer, W.M. Folkner, L.I. Gurvits, D.V. Johnston, D. Plettemeier, S.V. Pogrebenko, R.A. Preston, G.L. Tyler, The vertical profile of winds on Titan. Nature 438, 800-802 (2005). https://doi.org/ 10.1038/nature04060

J.E. Blamont et al., Implications of the VEGA balloon results for Venus atmospheric dynamics. Science 231, 1422-1425 (1986). https://doi.org/10.1126/science.231.4744.1422

S.J. Bolton, A. Adriani, V. Adumitroaie, M. Allison, J. Anderson, S. Atreya, J. Bloxham, S. Brown, J.E.P. Connerney, E. DeJong, W. Folkner, D. Gautier, D. Grassi, S. Gulkis, T. Guillot, C. Hansen, W.B. Hubbard, L. Iess, A. Ingersoll, M. Janssen, J. Jorgensen, Y. Kaspi, S.M. Levin, C. Li, J. Lunine, Y. Miguel, A. Mura, G. Orton, T. Owen, M. Ravine, E. Smith, P. Steffes, E. Stone, D. Stevenson, R. Thorne, J. Waite, D. Durante, R.W. Ebert, T.K. Greathouse, V. Hue, M. Parisi, J.R. Szalay, R. Wilson, Jupiter's interior and deep atmosphere: the initial pole-to-pole passes with the Juno spacecraft. Science 356, 821-825 (2017)

A. Bouchez, Seasonal trends in Titan's atmosphere: haze, winds, and clouds. PhD, California Institute of Technology (2004)

F.H. Busse, Thermal instabilities in rapidly rotating systems. J. Fluid Mech. 44, 441-460 (1970)

F.H. Busse, A simple model of convection in the Jovian atmosphere. Icarus 29, 255-260 (1976)

F.H. Busse, A model of mean zonal flows in the major planets. Geophys. Astrophys. Fluid Dyn. 23, 153-174 (1983). https://doi.org/10.1080/03091928308221746

F.H. Busse, Convection driven zonal flows and vortices in the major planets. Chaos 4(2), 123-134 (1994)

F.H. Busse, Convection flows in rapidly rotating spheres. Phys. Fluids 14, 1301-1314 (2002) 
H. Cao, D.J. Stevenson, Zonal flow magnetic field interaction in the semi-conducting region of giant planets. Icarus 296, 59-72 (2017)

K.L. Chan, H.G. Mayr, Numerical simulation of convectively generated vortices: application to the Jovian planets. Earth Planet. Sci. Lett. 371, 212-219 (2013)

R. Chemke, Y. Kaspi, The latitudinal dependence of atmospheric jet scales and macroturbulent energy cascades. J. Atmos. Sci. 72, 3891-3907 (2015)

J.Y.-K. Cho, L.M. Polvani, The emergence of jets and vortices in freely evolving, shallow-water turbulence on a sphere. Phys. Fluids 8, 1531-1552 (1996a)

J.Y.-K. Cho, L.M. Polvani, The morphogenesis of bands and zonal winds in the atmospheres on the giant outer planets. Science 273, 335-337 (1996b)

J.Y-K. Cho, I. Polichtchouk, H.Th. Thrastarson, Sensitivity and variability redux in hot-Jupiter flow simulations. Mon. Not. R. Astron. Soc. 454, 3423-3431 (2015)

D.S. Choi, A.P. Showman, R.H. Brown, Cloud features and zonal wind measurements of Saturn's atmosphere as observed by Cassini/VIMS. J. Geophys. Res. 114, E04007 (2009). https://doi.org/10.1029/ 2008JE003254

U.R. Christensen, Zonal flow driven by deep convection in the major planets. Geophys. Res. Lett. 28, 25532556 (2001)

R.T. Clancy, B.J. Sandor, G. Moriarty-Schieven, Circulation of the Venus upper mesosphere/lower thermosphere: Doppler wind measurements from 2001-2009 inferior conjunction, sub-millimeter CO absorption line observations. Icarus 217, 794-812 (2012). https://doi.org/10.1016/j.icarus.2011.05.021

J.E.P. Connerney, S. Kotsiaros, R.J. Oliversen, J.R. Espley, J.L. Joergensen, P.S. Joergensen, J.M.G. Merayo, M. Herceg, J. Bloxham, K.M. Moore, A new model of Jupiter's magnetic field from Juno's first nine orbits. Geophys. Res. Lett. 45, 2590-2596 (2018)

B.J. Conrath, F.M. Flasar, J.A. Pirraglia, P.J. Gierasch, G.E. Hunt, Thermal structure and dynamics of the Jovian atmosphere. II-visible cloud features. J. Geophys. Res. 86, 8769-8775 (1981)

C.S. Cooper, A.P. Showman, Dynamic meteorology at the photosphere of HD 209458b. Astrophys. J. 629, L45-L48 (2005)

C.C. Counselman, S.A. Gourevitch, R.W. King, G.B. Loriot, E.S. Ginsberg, Zonal and meridional circulation of the lower atmosphere of Venus determined by radio interferometry. J. Geophys. Res. 85, 8026-8030 (1980). https://doi.org/10.1029/JA085iA13p08026

A. Crespin, S. Lebonnois, S. Vinatier, B. Bézard, A. Coustenis, N.A. Teanby, R.K. Achterberg, P. Rannou, F. Hourdin, Diagnostics of Titan's stratospheric dynamics using Cassini/CIRS data and the IPSL General Circulation Model. Icarus 197, 556-571 (2008). https://doi.org/10.1016/j.icarus.2008.05.010

A.D. Del Genio, W.B. Rossow, Planetary-scale waves and the cyclic nature of cloud top dynamics on Venus. J. Atmos. Sci. 47, 293-318 (1990). https://doi.org/10.1175/1520-0469(1990)047<0293: PSWATC $>2.0 . \mathrm{CO} ; 2$

A.D. Del Genio, W. Zhou, T.P. Eichler, Equatorial superrotation in a slowly rotating GCM: implications for Titan and Venus. Icarus 101, 1-17 (1993)

A.D. Del Genio, J.M. Barbara, J. Ferrier, A.P. Ingersoll, R.A. West, A.R. Vasavada, J. Spitale, C.C. Porco, Saturn eddy momentum fluxes and convection: first estimates from Cassini images. Icarus 189, 479-492 (2007)

B.O. Demory et al., A map of the large day-night temperature gradient of a super-Earth exoplanet. Nature 532, 207-209 (2016). https://doi.org/10.1038/nature17169

J.R. dias Pinto, J.L. Mitchell, Atmospheric superrotation in an idealized GCM: parameter dependence of the eddy response. Icarus 238, 93 (2014)

W. Dietrich, C.A. Jones, Anelastic spherical dynamos with radially variable electrical conductivity. Icarus 305, 15 (2018)

I. Dobbs-Dixon, E. Agol, Three-dimensional radiative-hydrodynamical simulations of the highly irradiated short-period exoplanet HD 189733b. Mon. Not. R. Astron. Soc. 435, 3159-3168 (2013)

I. Dobbs-Dixon, D.N.C. Lin, Atmospheric dynamics of short-period extrasolar gas giant planets. I. Dependence of nightside temperature on opacity. Astrophys. J. 673, 513-525 (2008)

T.E. Dowling, A.P. Ingersoll, Potential vorticity and layer thickness variations in the flow around Jupiter's great red spot and white oval BC. J. Atmos. Sci. 45, 1380-1396 (1988)

T.E. Dowling, A.P. Ingersoll, Jupiter's great red spot as a shallow water system. J. Atmos. Sci. 46, 3256-3278 (1989)

K. Duer, E. Galanti, Y. Kaspi, Analysis of Jupiter's deep jets combining Juno gravity and time-varying magnetic field measurements. Astrophys. J. Lett. 879, L22 (2019)

S.G. Edgington, L.J. Spilker, Cassini's grand finale. Nat. Geosci. 9, 472-473 (2016)

A. Edson, S. Lee, P. Bannon, J.F. Kasting, D. Pollard, Atmospheric circulations of terrestrial planets orbiting low-mass stars. Icarus 212, 1-13 (2011) 
V. Eymet, R. Fournier, J.-L. Dufresne, S. Lebonnois, F. Hourdin, M.A. Bullock, Net-exchange parameterization of the thermal infrared radiative transfer in Venus' atmosphere. J. Geophys. Res. 114, E11008 (2009). https://doi.org/10.1029/2008JE003276

B.F. Farrell, P. Ioannou, Emergence of jets from turbulence in the shallow-water equations on an equatorial beta plane. J. Atmos. Sci. 66, 3197-3207 (2009)

F.M. Flasar, B.J. Conrath, Titan's stratospheric temperatures: a case for dynamical inertia? Icarus 85, 346-354 (1990)

F.M. Flasar, R.E. Samuelson, B.J. Conrath, Titan's atmosphere: temperature and dynamics. Nature 292, 693698 (1981)

F.M. Flasar et al., Titan's atmospheric temperatures, winds, and composition. Science 308, 975-978 (2005)

E. Flowers, M. Brogi, E. Rauscher, E.M-R. Kempton, A. Chiavassa, The high-resolution transmission spectrum of HD 189733b interpreted with atmospheric Doppler shifts from three-dimensional general circulation models. Astrophys. J. 157, 209 (2019)

D.C. Fritts, M.J. Alexander, Gravity wave dynamics and effects in the middle atmosphere. Rev. Geophys. 41, 1003 (2003). https://doi.org/10.1029/2001RG000106

T. Fukuhara et al., Large stationary gravity wave in the atmosphere of Venus. Nat. Geosci. 10, 85-88 (2017). https://doi.org/10.1038/ngeo2873

E. Galanti, H. Cao, Y. Kaspi, Constraining Jupiter's internal flows using Juno magnetic and gravity measurements. Geophys. Res. Lett. 44, 8173-8181 (2017)

E. Galanti, Y. Kaspi, Y. Miguel, T. Guillot, D. Durante, P. Racioppa, L. Iess, Saturn's deep atmospheric flows revealed by the Cassini grand finale gravity measurements. Geophys. Res. Lett. 46, 616-624 (2019)

I. Garate-Lopez, S. Lebonnois, Latitudinal variation of clouds' structure responsible for Venus cold collar. Icarus 314, 1-11 (2018). https://doi.org/10.1016/j.icarus.2018.05.011

C.I. Garfinkel, I. Fouxon, O. Shamir, N. Paldor, Classification of eastward propagating waves on the spherical Earth. Q. J. R. Meteorol. Soc. 143, 1554-1564 (2017)

T. Gastine, J. Wicht, J.M. Aurnou, Zonal flow regimes in rotating anelastic spherical shells: an application to giant planets. Icarus 225, 156-172 (2013)

P. Gierasch, Meridional circulation and the maintenance of the Venus atmospheric rotation. J. Atmos. Sci. 32, 1038-1044 (1975)

P.J. Gierasch, J.A. Magalhaes, B.J. Conrath, Zonal mean properties of Jupiter's upper troposphere from Voyagerinfrared observations. Icarus 67, 456-483 (1986)

A.E. Gill, Some simple solutions for heat-induced tropical circulation. Q. J. R. Meteorol. Soc. 106, 447-462 (1980)

D.A. Gorinov, I.V. Khatuntsev, L.V. Zasova, A.V. Turin, G. Piccioni, Circulation of Venusian atmosphere at 90-110 km based on apparent motions of the $\mathrm{O}_{2} 1.27 \mu \mathrm{m}$ nightglow from VIRTIS-M (Venus Express) data. Geophys. Res. Lett. 45, 2554-2562 (2018). https://doi.org/10.1002/2017GL076380

R. Greeley, K. Bender, P.E. Thomas, G. Schubert, D. Limonadi, C.M. Weitz, Wind-related features and processes on Venus: summary of Magellan results. Icarus 115, 399-420 (1995)

I. Guendelman, Y. Kaspi, An axisymmetric limit for the width of the Hadley Cell on planets with large obliquity and long seasonality. Geophys. Res. Lett. 45, 13,213-13,221 (2018)

I. Guendelman, Y. Kaspi, Atmospheric dynamics on terrestrial planets: the seasonal response to changes in orbital, rotational, and radiative timescales. Astrophys. J. 881, 67 (2019)

S. Guerlet, A. Spiga, M. Sylvestre et al., Global climate modeling of Saturn's atmosphere. Part I: evaluation of the radiative transfer model. Icarus 238, 110-124 (2014)

T. Guillot, A. Burrows, W.B. Hubbard, J.I. Lunine, D. Saumon, Giant planets at small orbital distances. Astrophys. J. 459, L35-L38 (1996)

R.A. Hanel, B.J. Conrath, V.G. Kunde, J.C. Pearl, J.A. Pirraglia, Albedo, internal heat flux, and energy balance of Saturn. Icarus 53, 262-285 (1983)

Y.Y. Hayashi, Rossby waves and jets in two-dimensional decaying turbulence on a rotating sphere. J. Atmos. Sci. 64, 4246-4269 (2007)

M. Heimpel, J. Aurnou, J. Wicht, Simulation of equatorial and high-latitude jets on Jupiter in a deep convection model. Nature 438, 193-196 (2005)

M. Heimpel, T. Gastine, J. Wicht, Simulation of deep seated zonal jets and shallow vortices in gas giant atmospheres. Nat. Geosci. 9, 19-23 (2016)

I.M. Held, A.Y. Hou, Nonlinear axially symmetric circulations in a nearly inviscid atmosphere. J. Atmos. Sci. 37, 515-533 (1980). https://doi.org/10.1175/1520-0469(1980)037<0515:NASCIA >2.0.CO;2

K. Heng, A.P. Showman, Atmospheric dynamics of hot exoplanets. Annu. Rev. Earth Planet. Sci. 43, 509-540 (2015)

K. Heng, S.S. Vogt, Gliese 581g as a scaled-up version of Earth: atmospheric circulation simulations. Mon. Not. R. Astron. Soc. 415, 2145-2157 (2011) 
K. Heng, K. Menou, P.J. Phillipps, Atmospheric circulation of tidally locked exoplanets: a suite of benchmark tests for dynamical solvers. Mon. Not. R. Astron. Soc. 413, 2380-2402 (2011a)

K. Heng, D.M.W. Frierson, P.J. Phillipps, Atmospheric circulation of tidally locked exoplanets: II. Dual-band radiative transfer and convective adjustment. Mon. Not. R. Astron. Soc. 418, 2669-2696 (2011b)

A. Herrnstein, T.E. Dowling, Effect of topography on the spin-up of a Venus atmospheric model. J. Geophys. Res. 112, E04S08 (2007). https://doi.org/10.1029/2006JE002804

R. Hide, Dynamics of the atmospheres of the major planets, with an appendix on the viscous boundary layer at the rigid bounding surface of an electrically-conducting rotating fluid in the presence of a magnetic field. J. Atmos. Sci. 26, 841-853 (1969). https://doi.org/10.1175/15200469(1969)026<0841:DOTAOT>2.0.CO;2

J.L. Hollingsworth, R.E. Young, G. Schubert, C. Covey, A.S. Grossman, A simple-physics global circulation model for Venus: sensitivity assessments of atmospheric superrotation. Geophys. Res. Lett. 34, L05202 (2007). https://doi.org/10.1029/2006GL028567

J.R. Holton, G.J. Hakim, An Introduction to Dynamic Meteorology, 5th edn. (Academic Press, San Diego, 2013). $532 \mathrm{pp}$.

T. Horinouchi, S.-Y. Murakami, T. Satoh, J. Peralta, K. Ogohara, T. Kouyama, T. Imamura, H. Kashimura, S.S. Limaye, K. McGouldrick, M. Nakamura, T.M. Sato, K.-I. Sugiyama, M. Takagi, S. Watanabe, M. Yamada, A. Yamazaki, E.F. Young, Equatorial jet in the lower to middle cloud layer of Venus revealed by Akatsuki. Nat. Geosci. 10, 646-651 (2017). https://doi.org/10.1038/ngeo3016

T. Horinouchi, T. Kouyama, Y.J. Lee, S.-Y. Murakami, K. Ogohara, M. Takagi, T. Imamura, K. Nakajima, J. Peralta, A. Yamazaki, M. Yamada, S. Watanabe, Mean winds at the cloud top of Venus obtained from two-wavelength UV imaging by Akatsuki. Earth Planets Space 70, 10 (2018). https://doi.org/ 10.1186/s40623-017-0775-3

T. Horinouchi et al., How waves and turbulence maintain the super-rotation of Venus' atmosphere. Science 368, 405-409 (2020). https://doi.org/10.1126/science.aaz4439

S.M. Hörst, Titan's atmosphere and climate. J. Geophys. Res. 122, 432-482 (2017). https://doi.org/10.1002/ 2016JE005240

F. Hourdin, O. Talagrand, R. Sadourny, R. Courtin, D. Gautier, C.P. McKay, Numerical simulation of the general circulation of the atmosphere of Titan. Icarus 117, 358-374 (1995)

F. Hourdin, S. Lebonnois, D. Luz, P. Rannou, Titan's stratospheric composition driven by condensation and dynamics. J. Geophys. Res. 109, E12005 (2004). https://doi.org/10.1029/2004JE002282

Y. Hu, J. Yang, Role of ocean heat transport in climates of tidally locked exoplanets around M dwarf stars. Proc. Natl. Acad. Sci. 111, 629-634 (2014)

H.-P. Huang, W.A. Robinson, Two-dimensional turbulence and persistent zonal jets in a global barotropic model. J. Atmos. Sci. 55, 611-632 (1998)

W.B. Hubbard et al., The occultation of 28 Sgr by Titan. Astron. Astrophys. 269, 541-563 (1993)

R. Hueso, J. Peralta, I. Garate-Lopez, T.V. Bandos, A. Sánchez-Lavega, Six years of Venus winds at the upper cloud level from UV, visible and near infrared observations from VIRTIS on Venus Express. Planet. Space Sci. 113, 78-99 (2015). https://doi.org/10.1016/j.pss.2014.12.010

L. Iess, W.M. Folkner, D. Durante, M. Parisi, Y. Kaspi, E. Galanti, T. Guillot, W.B. Hubbard, D.J. Stevenson, J.D. Anderson, D.R. Buccino, L.G. Casajus, A. Milani, R. Park, P. Racioppa, D. Serra, P. Tortora, M. Zannoni, H. Cao, R. Helled, J.I. Lunine, Y. Miguel, B. Militzer, S. Wahl, J.E.P. Connerney, S.M. Levin, S.J. Bolton, Measurement of Jupiter's asymmetric gravity field. Nature 555, 220-222 (2018)

L. Iess, B. Militzer, Y. Kaspi, P. Nicholson, D. Durante, P. Racioppa, A. Anabtawi, E. Galanti, W.B. Hubbard, M.J. Mariani, P. Tortora, S. Wahl, M. Zannoni, Measurement and implications of Saturn's gravity field and ring mass. Science 364, 1052 (2019)

K. Ikeda, Development of Radiative Transfer Model for Venus Atmosphere and Simulation of Superrotation Using a General Circulation Model. PhD thesis, University of Tokyo (2011)

M. Imai, T. Kouyama, Y. Takahashi, A. Yamazaki, S. Watanabe, M. Yamada, T. Imamura, T. Satoh, M. Nakamura, S. Murakami, K. Ogohara, T. Horinouchi, Planetary-scale variations in winds and UV brightness at the Venusian cloud top: periodicity and temporal evolution. J. Geophys. Res. 124(10), 2635-2659 (2019). https://doi.org/10.1029/2019JE006065

T. Imamura, Momentum balance of the Venusian midlatitude mesosphere. J. Geophys. Res. 102, 6615-6620 (1997)

T. Imamura, G.L. Hashimoto, Venus cloud formation in the meridional circulation. J. Geophys. Res. 103, 31349-31366 (1998)

T. Imamura, M. Miyamoto, H. Ando, B. Häusler, M. Pätzold, S. Tellmann, T. Tsuda, Y. Aoyama, Y. Murata, H. Takeuchi, A. Yamazaki, T. Toda, A. Tomiki, Fine vertical structures at the cloud heights of Venus revealed by radio holographic analysis of Venus Express and Akatsuki radio occultation data. J. Geophys. Res. 123, 2151-2161 (2018). https://doi.org/10.1029/2018JE005627 
A.P. Ingersoll, P.J. Gierasch, D. Banfield, A.R. Vasavada, the Galileo imaging team, Moist convection as an energy source for the large-scale motions in Jupiter's atmosphere. Nature 403, 630-632 (2000)

K. Ishioka, Asymmetrization mechanism of jet profiles in decaying $\beta$-plane turbulence. J. Atmos. Sci. 64, 3354-3361 (2007). https://doi.org/10.1175/JAS4011.1

C.A. Jones, K.M. Kuzanyan, Compressible convection in the deep atmospheres of giant planets. Icarus 204, 227-238 (2009)

M.M. Joshi, R.M. Haberle, R.T. Reynolds, Simulations of the atmospheres of synchronously rotating terrestrial planets orbiting M dwarfs: conditions for atmospheric collapse and the implications for habitability. Icarus 129, 450-465 (1997)

H. Kashimura, S. Yoden, Regime diagrams of solutions in an idealized quasi-axisymmetric model for superrotation of planetary atmospheres. J. Meteorol. Soc. Jpn. 93, 309-326 (2015) https://doi.org/10.2151/ jmsj.2015-017

H. Kashimura et al., Planetary-scale streak structure reproduced in high-resolution simulations of the Venus atmosphere with a low-stability layer. Nat. Commun. 10, 23 (2019). https://doi.org/10.1038/ s41467-018-07919-y

Y. Kaspi, Inferring the depth of the zonal jets on Jupiter and Saturn from odd gravity harmonics. Geophys. Res. Lett. 40, 676-680 (2013)

Y. Kaspi, G.R. Flierl, Formation of jets by baroclinic instability on gas planet atmospheres. J. Atmos. Sci. 64, 3177-3194 (2007)

Y. Kaspi, G.R. Flierl, A.P. Showman, The deep wind structure of the giant planets: results from an anelastic general circulation model. Icarus 202, 525-542 (2009)

Y. Kaspi, W.B. Hubbard, A.P. Showman, G.R. Flierl, Gravitational signature of Jupiter's internal dynamics. Geophys. Res. Lett. 37, 01204 (2010)

Y. Kaspi et al., Jupiter's atmospheric jet streams extend thousands of kilometres deep. Nature 555, 223-226 (2018). https://doi.org/10.1038/nature25793

Y. Kaspi, E. Galanti, A.P. Showman, D.J. Stevenson, T. Guillot, L. Iess, S.J. Bolton, Comparison of the deep atmospheric dynamics of Jupiter and Saturn in light of the Juno and Cassini gravity measurements. Space Sci. Rev. (2020), in press. https://doi.org/10.1007/s11214-020-00705-7

T. Kataria, A.P. Showman, J.J. Fortney, K.B. Stevenson, M.R. Line, L. Kreidberg, J.L. Bean, J-M. Desert, The atmospheric circulation of the hot Jupiter WASP-43b: comparing three-dimensional models to spectrophotometric data. Astrophys. J. 801, 86 (2015)

D. Kataria, D.K. Sing, N.K. Lewis, C. Visscher, A.P. Showman, J.J. Fortney, M.S. Marley, The atmospheric circulation of a nine-hot-Jupiter sample: probing circulation and chemistry over a wide phase space. Astrophys. J. 821, 9 (2016)

E.M-R. Kempton, E. Rauscher, Constraining high-speed winds in exoplanet atmospheres through observations of anomalous Doppler shifts during transit. Astrophys. J. 751, 117 (2012)

V.V. Kerzhanovich, M.Y. Marov, The atmospheric dynamics of Venus according to Doppler measurements by the Venera entry probes, in Venus I, ed. by D.M. Hunten, L. Colin, T.M. Donahue, V.I. Moroz (University of Arizona Press, Tucson, 1983), pp. 766-778

I.V. Khatuntsev, M.V. Patsaeva, D.V. Titov, N.I. Ignatiev, A.V. Turin, S.S. Limaye, W.J. Markiewicz, M. Almeida, Th. Roatsch, R. Moissl, Cloud level winds from the Venus Express Monitoring Camera imaging. Icarus 226, 140-158 (2013)

I.V. Khatuntsev, M.V. Patsaeva, D.V. Titov, N.I. Ignatiev, A.V. Turin, A.A. Fedorova, W.J. Markiewicz, Winds in the middle cloud deck from the near-IR imaging by the Venus Monitoring Camera onboard Venus Express. J. Geophys. Res. 122, 2312-2327 (2017)

T. Kitahara, T. Imamura, T.M. Sato, A. Yamazaki, Y.J. Lee, M. Yamada et al., Stationary features at the cloud top of Venus observed by Ultraviolet Imager onboard Akatsuki. J. Geophys. Res. 124, 1266-1281 (2019). https://doi.org/10.1029/2018JE005842

H.A. Knutson et al., A map of the day-night contrast of the extrasolar planet HD 189733b. Nature 447, 183-186 (2007)

T. Komacek, A.P. Showman, X. Tan, Atmospheric circulation of hot Jupiters: dayside-nightside temperature differences. II. Comparison with observations. Astrophys. J. 835, 198 (2017)

T. Kostiuk, K.E. Fast, T.A. Livengood, T. Hewagama, J. Goldstein, F. Espenak, D. Buhl, Direct measurements of winds on Titan. Geophys. Res. Lett. 28, 2361-2364 (2001)

T. Kostiuk, T.A. Livengood, T. Hewagama, G. Sonnabend, K.E. Fast, K. Murakawa, A.T. Tokunaga, J. Annen, D. Buhl, F. Schmülling, Titan's stratospheric zonal wind, temperature, and ethane abundance a year prior to Huygens insertion. Geophys. Res. Lett. 32, L22205 (2005)

T. Kostiuk, T.A. Livengood, G. Sonnabend, K.E. Fast, T. Hewagama, K. Murakawa, A.T. Tokunaga, J. Annen, D. Buhl, F. Schmülling, D. Luz, O. Witasse, Stratospheric global winds on Titan at the time of Huygens descent. J. Geophys. Res. 111, E07S03 (2006) 
T. Kostiuk, T. Hewagama, K.E. Fast, T.A. Livengood, J. Annen, D. Buhl, G. Sonnabend, F. Schmülling, J.D. Delgado, R. Achterberg, High spectral resolution infrared studies of Titan: winds, temperature, and composition. Planet. Space Sci. 58, 1715-1723 (2010). https://doi.org/10.1016/j.pss.2010.08.004

T. Kouyama, T. Imamura, M. Nakamura, T. Satoh, Y. Futaana, Horizontal structure of planetary-scale waves at the cloud top of Venus deduced from Galileo SSI images with an improved cloud-tracking technique. Planet. Space Sci. 60, 207-216 (2012). https://doi.org/10.1016/j.pss/2011.08.008

T. Kouyama, T. Imamura, M. Nakamura, T. Satoh, Y. Futaana, Long-term variation in the cloud-tracked zonal velocities at the cloud top of Venus deduced from Venus Express VMC images. J. Geophys. Res. 118, 37-46 (2013). https://doi.org/10.1029/2011JE004013

T. Kouyama, T. Imamura, M. Nakamura, T. Satoh, Y. Futaana, Vertical propagation of planetary-scale waves in variable background winds in the upper cloud region of Venus. Icarus 248, 560-568 (2015). https://doi.org/10.1016/j.icarus.2014.07.011

T. Kouyama et al., Topographical and local time dependence of large stationary gravity waves observed at the cloud top of Venus. Geophys. Res. Lett. 44, 12,098-12,105 (2017). https://doi.org/10.1002/ 2017GL075792

T. Kouyama, M. Taguchi, T. Fukuhara, T. Imamura, T. Horinouchi, T.M. Sato, S. Murakami, G.L. Hashimoto, Y.J. Lee, M. Futaguchi, T. Yamada, M. Akiba, T. Satoh, M. Nakamura, Global structure of thermal tides in the upper cloud layer of Venus revealed by LIR on board Akatsuki. Geophys. Res. Lett. 46(16), 9457-9465 (2019). https://doi.org/10.1029/2019GL083820

I. Kraucunas, D.L. Hartmann, Equatorial superrotation and the factors controlling the zonal-mean zonal winds in the tropical upper troposphere. J. Atmos. Sci. 62, 371-389 (2005). https://doi.org/ 10.1175/JAS-3365.1

S. Lebonnois, F. Hourdin, P. Rannou, The coupling of winds, aerosols and photochemistry in Titan's atmosphere. Philos. Trans. R. Soc. Lond. A 367, 665-682 (2009). https://doi.org/10.1098/rsta.2008.0243

S. Lebonnois, F. Hourdin, V. Eymet, A. Crespin, R. Fournier, F. Forget, Super-rotation of Venus' atmosphere analysed with a full general circulation model. J. Geophys. Res. 115, E06006 (2010). https://doi.org/10.1029/2009JE003458

S. Lebonnois, C. Covey, A. Grossman, H. Parish, G. Schubert, R. Walterscheid, P. Lauritzen, C. Jablonowski, Angular momentum budget in general circulation models of superrotating atmospheres: a critical diagnostic. J. Geophys. Res. 117, E12004 (2012a). https://doi.org/10.1029/2012JE004223

S. Lebonnois, J. Burgalat, P. Rannou, B. Charnay, Titan Global Climate Model: new 3-dimensional version of the IPSL Titan GCM. Icarus 218, 707-722 (2012b). https://doi.org/10.1016/j.icarus.2011.11.032

S. Lebonnois, C. Lee, M. Yamamoto, J. Dawson, S.R. Lewis, J. Mendonca, P.L. Read, H. Parish, G. Schubert, L. Bengtsson, D. Grinspoon, S. Limaye, H. Schmidt, H. Svedhem, D. Titov, Models of Venus atmosphere, in Towards Understanding the Climate of Venus: Application of Terrestrial Models to Our Sister Planet, ed. by L. Bengtsson, R.-M. Bonnet, D. Grinspoon, S. Koumoutsaris, S. Lebonnois, D. Titov. ISSI Scientific Report Series, vol. 11 (Springer, Netherlands, 2013), pp. 129-156

S. Lebonnois, F.M. Flasar, T. Tokano, C.E. Newman, The general circulation of Titan's lower and middle atmosphere, in Titan: Interior, Surface, Atmosphere and Space Environment, ed. by I. Mueller-Wodarg, C. Griffith, E. Lellouch, T. Cravens (Cambridge University Press, United Kingdom, 2014), pp. 122-157

S. Lebonnois, N. Sugimoto, G. Gilli, Wave analysis in the atmosphere of Venus below 100-km altitude, simulated by the LMD Venus GCM. Icarus 278, 38-51 (2016). https://doi.org/10.1016/j.icarus.2016.06.004

J. Leconte, F. Forget, B. Charnay, R. Wordsworth, F. Selsis, E. Millour, A. Spiga, 3D climate modeling of close-in land planets: circulation patterns, climate moist bistability, and habitability. Astron. Astrophys. 554, A69 (2013)

S. Lee, Why are the climatological zonal winds easterly in the equatorial upper troposphere? J. Atmos. Sci. 56, 1353-1363 (1999). https://doi.org/10.1175/1520-0469(1999)056<1353:WATCZW>2.0.CO;2

C. Lee, M.I. Richardson, A general circulation model ensemble study of the atmospheric circulation of Venus. J. Geophys. Res. 115, E04002 (2010). https://doi.org/10.1029/2009JE003490

C. Lee, S.R. Lewis, P.L. Read, A numerical model of the atmosphere of Venus. Adv. Space Res. 36, 21422145 (2005). https://doi.org/10.1016/j.asr.2005.03.120

C. Lee, S.R. Lewis, P.L. Read, Super-rotation in a Venus general circulation model. J. Geophys. Res. 112, E04S11 (2007). https://doi.org/10.1029/2006JE002874

Y.J. Lee, D.V. Titov, N.I. Ignatiev, S. Tellmann, M. Pätzold, G. Piccioni, The radiative forcing variability caused by the changes of the upper cloud vertical structure in the Venus mesosphere. Planet. Space Sci. 113-114, 298-308 (2015). https://doi.org/10.1016/j.pss.2014.12.006

S.R. Lewis, C. Lee, P.L. Read, A Venus atmospheric general circulation model for Venus Express, in European Planetary Science Congress, Berlin, Germany, 18-22 Sept. 2006 (2006)

N.K. Lewis, A.P. Showman, J.J. Fortney, M.S. Marley, R.S. Freedman, K. Lodders, Atmospheric circulation of eccentric hot Neptune GJ436b. Astrophys. J. 720, 344-356 (2010) 
S.R. Lewis, J. Dawson, S. Lebonnois, M. Yamamoto, Modelling efforts, in Towards Understanding the Climate of Venus, ed. by L. Bengtsson, R.-M. Bonnet, D. Grinspoon, S. Koumoutsaris, S. Lebonnois, D. Titov. ISSI Scientific Report Series, vol. 11 (Springer, Netherlands, 2013), pp. 111-128

L. Li et al., Less absorbed solar energy and more internal heat for Jupiter. Nat. Commun. 9, 3709 (2018). https://doi.org/10.1038/s41467-018-06107-2

Y. Lian, A.P. Showman, Deep jets on gas-giant planets. Icarus 194, 597-615 (2008)

Y. Lian, A.P. Showman, Generation of equatorial jets by large-scale latent heating on the giant planets. Icarus 207, 373-393 (2010)

S.S. Limaye, Venus atmospheric circulation: known and unknown. J. Geophys. Res. 112, E04S09 (2007). https://doi.org/10.1029/2006JE002814

S.S. Limaye, V.E. Suomi, Cloud motions on Venus: global structure and organization. J. Atmos. Sci. 38, 1220-1235 (1981)

S.S. Limaye, C. Grassotti, M.J. Kuetemeyer, Venus: cloud level circulation during 1982 as determined from Pioneer cloud photopolarimeter images. I-time and zonally averaged circulation. Icarus 73, 193-211 (1988a)

S.S. Limaye, et al., Venus: cloud level circulation during 1982 as determined from Pioneer cloud photopolarimeter images. II-solar longitude dependent circulation. Icarus 73, 212-226 (1988b)

V.M. Linkin, V.V. Kerzhanovich, A.N. Lipatov, K.M. Pichkadze, A.A. Shurupov, A.V. Tertebrashvili, A.P. Ingersoll, D. Crisp, A.W. Grossman, R.E. Young, A. Seiff, B. Ragent, J.E. Blamont, L.S. Elson, R. Preston, VEGA balloon dynamics and vertical winds in the Venus middle cloud region. Science $\mathbf{2 3 1}$, 1417-1419 (1986)

J. Liu, T. Schneider, Mechanisms of jet formation on the giant planets. J. Atmos. Sci. 67, 3652-3672 (2010)

J. Liu, P.M. Goldreich, D.J. Stevenson, Constraints on deep-seated zonal winds inside Jupiter and Saturn. Icarus 196, 653-664 (2008)

J.M. Lora, J.I. Lunine, J.L. Russell, GCM simulations of Titan's middle and lower atmosphere and comparison to observations. Icarus 250, 516-528 (2015). https://doi.org/10.1016/j.icarus.2014.12.030

T. Louden, P.J. Wheatley, Spatially resolved eastward wind and rotation of HD 189733b. Astrophys. J. Lett. 814, L24 (2015)

D. Luz, F. Hourdin, Latitudinal transport by barotropic waves in Titan's stratosphere. I. General properties from a horizontal shallow-water model. Icarus 166, 328-342 (2003)

D. Luz, F. Hourdin, P. Rannou, S. Lebonnois, Latitudinal transport by barotropic waves in Titan's stratosphere. II. Results from a coupled dynamics-microphysics-photochemistry GCM. Icarus 166, 343-358 (2003)

D. Luz, T. Civeit, R. Courtin, J.-P. Lebreton, D. Gautier, P. Rannou, A. Kaufer, O. Witasse, L. Lara, F. Ferri, Characterization of zonal winds in the stratosphere of Titan with UVES. Icarus 179, 497-510 (2005)

D. Luz, T. Civeit, R. Courtin, J.-P. Lebreton, D. Gautier, O. Witasse, A. Kaufer, F. Ferri, L. Lara, T. Livengood, T. Kostiuk, Characterization of zonal winds in the stratosphere of Titan with UVES: 2. Observations coordinated with the Huygens Probe entry. J. Geophys. Res. 111, E08S90 (2006). https://doi.org/10.1029/2005JE002617

P. Machado, T. Widemann, D. Luz, J. Peralta, Wind circulation regimes at Venus' cloud tops: ground-based Doppler velocimetry using CFHT/ESPaDOnS and comparison with simultaneous cloud tracking measurements using VEx/VIRTIS in February 2011. Icarus 243, 249-263 (2014)

P. Machado, T. Widemann, J. Peralta, R. Gonçalves, J.-F. Donati, D. Luz, Venus cloud-tracked and Doppler velocimetry winds from CFHT/ESPaDOnS and Venus Express/VIRTIS in April 2014. Icarus 285, 8-26 (2017). https://doi.org/10.1016/j.icarus.2016.12.017

A.J. Manifori, W.R. Young, Slow evolution of zonal jets on the beta plane. J. Atmos. Sci. 56, 784-800 (1999)

Y. Matsuda, Dynamics of the four-day circulation in the Venus atmosphere. J. Meteorol. Soc. Jpn. 58, 443470 (1980)

N.J. Mayne et al., The unified model, a fully-compressible, non-hydrostatic, deep atmosphere global circulation model, applied to hot Jupiters. ENDGame for a HD 209458b test case. Astron. Astrophys. 561, A1 (2014)

N.J. Mayne et al., Results from a set of three-dimensional numerical experiments of a hot Jupiter atmosphere. Astron. Astrophys. 604, A79 (2017)

H.G. Mayr, I. Harris, Quasi-axisymmetric circulation and super-rotation in planetary atmospheres. Astron. Astrophys. 121, 124-136 (1983)

C.P. McKay, J.B. Pollack, R. Courtin, The thermal structure of Titan's atmosphere. Icarus 80, 23-53 (1989)

J.M. Mendonca, P.L. Read, Exploring the Venus global super-rotation using a comprehensive general circulation model. Planet. Space Sci. 134, 1-18 (2016). https://doi.org/10.1016/j.pss.2016.09.001

J.M. Mendonca, S-M. Tsai, M. Malik, S.L. Grimm, K. Heng, Three-dimensional circulation driving chemical disequilibrium in WASP-43b. Astrophys. J. 869, 107 (2018)

K. Menou, Atmospheric circulation and composition of GJ1214b. Astrophys. J. Lett. 744, L16 (2012) 
K. Menou, E. Rauscher, Atmospheric circulation of hot Jupiters: a shallow three-dimensional model. Astrophys. J. 700, 887-897 (2009)

T.M. Merlis, T. Schneider, Atmospheric dynamics of Earth-like tidally locked aquaplanets. J. Adv. Model. Earth Syst. 2, 13 (2010)

M.S. Miesch, A.S. Brun, J. Toomre, Solar differential rotation influenced by latitudinal entropy variations in the tachocline. Astrophys. J. 641, 618-625 (2006)

J.L. Mitchell, G.K. Vallis, The transition to superrotation in terrestrial atmospheres. J. Geophys. Res. 115, E12008 (2010)

J.L. Mitchell, R.T. Pierrehumbert, D.M.W. Frierson, R. Caballero, The dynamics behind Titan's methane cloud. Proc. Natl. Acad. Sci. 103, 18421-18426 (2006). https://doi.org/10.1073/pnas.0605074103

J.L. Mitchell, M. Adamkovics, R. Caballero, E.P. Turtle, Locally enhanced precipitation organized by planetary-scale waves on Titan. Nat. Geosci. 4, 589-592 (2011). https://doi.org/10.1038/ngeo1219

J.L. Mitchell, G.K. Vallis, S.F. Potter, Effects of the seasonal cycle on superrotation in planetary atmospheres. Astrophys. J. 787, 23 (2014)

K.M. Moore, H. Cao, J. Bloxham, D.J. Stevenson, J.E.P. Connerney, S.J. Bolton, Time variation of Jupiter's internal magnetic field consistent with zonal wind advection. Nat. Astron. 3, 730-735 (2019)

R. Moreno, A. Marten, T. Hidayat, Interferometric measurements of zonal winds on Titan. Astron. Astrophys. 437, 319-328 (2005)

V.I. Moroz, L.V. Zasova, VIRA-2: a review of inputs for updating the Venus international reference atmosphere. Adv. Space Res. 19, 1191-1201 (1997)

M. Nakamura et al., AKATSUKI returns to Venus. Earth Planets Space 68, 75 (2016). https://doi.org/ 10.1186/s40623-016-0457-6

T. Nasuno, Equatorial mean zonal wind in a global nonhydrostatic aquaplanet experiment. J. Meteorol. Soc. Jpn. 86A, 219-236 (2008). https://doi.org/10.2151/jmsj.86A.219

M. Newman, G. Schubert, A.J. Kliore, I.R. Patel, Zonal winds in the middle atmosphere of Venus from Pioneer Venus radio occultation data. J. Atmos. Sci. 41, 1901-1913 (1984). https://doi.org/ 10.1175/1520-0469(1984)041<1901:ZWITMA>2.0.CO;2

C.E. Newman, C. Lee, Y. Lian, M.I. Richardson, A.D. Toigo, Stratospheric superrotation in the TitanWRF model. Icarus 213, 636-654 (2011). https://doi.org/10.1016/j.icarus.2011.03.025

M.E. O’Neill, M.E. Kaspi, Slantwise convection on fluid planets. Geophys. Res. Lett. 43, 10611-10620 (2016)

G.S. Orton, B.M. Fisher, K.H. Baines, S.T. Stewart, A.J. Friedson, J.L. Ortiz, M. Marinova, M. Ressler, A. Dayal, v. Hoffmann, v. Hora, S. Hinkley, V. Krishnan, M. Masanovic, J. Tesic, A. Tziolas, K.C. Parija, Characteristics of the Galileo probe entry site from Earth-based remote sensing observations. J. Geophys. Res. 103, 22791-22814 (1998)

R.L. Panetta, Zonal jets in wide baroclinically unstable regions: persistence and scale selection. J. Atmos. Sci. 50, 2073-2106 (1993)

V. Parmentier, A.P. Showman, Y. Lian, 3D mixing in hot Jupiter atmospheres I. Application to the day/night cold trap in HD 209458b. Astron. Astrophys. 558, A91 (2013)

V. Parmentier, J.J. Fortney, A.P. Showman, C. Morley, M.S. Marley, Transitions in the cloud compositions of hot Jupiters. Astrophys. J. 828, 22 (2016)

M.V. Patsaeva, I.V. Khatuntsev, L.V. Zasova, A. Hauchecorne, D.V. Titov, J.-L. Bertaux, Solar-related variations of the cloud top circulation above Aphrodite Terra from VMC/Venus Express wind fields. J. Geophys. Res. 124, 1864-1879 (2019)

J. Pedlosky, Geophysical Fluid Dynamics (Springer, Berlin, 1987), p. 710

J. Peralta, R. Hueso, A. Sánchez-Lavega, A reanalysis of Venus winds at two cloud levels from Galileo SSI images. Icarus 190, 469-477 (2007). https://doi.org/10.1016/j.icarus.2007.03.028

J. Peralta, R. Hueso, A. Sánchez-Lavega, G. Piccioni, O. Lanciano, P. Drossart, Characterization of mesoscale gravity waves in the upper and lower clouds of Venus from VEX-VIRTIS images. J. Geophys. Res. 113, E00B18 (2008). https://doi.org/10.1029/2008JE003185

J. Peralta, D. Luz, D.L. Berry, C.C.C. Tsang, A. Sánchez-Lavega, R. Hueso, G. Piccioni, P. Drossart, Solar migrating atmospheric tides in the winds of the polar region of Venus. Icarus 220, 958-970 (2012)

J. Peralta, A. Sánchez-Lavega, M.A. Lopez-Valverde, D. Luz, P. Machado, Venus's major cloud feature as an equatorially-trapped wave distorted by the wind. Geophys. Res. Lett. 42, 705-711 (2015). https:// doi.org/10.1002/2014GL062280

J. Peralta, R. Hueso, A. Sánchez-Lavega, Y.J. Lee, A. García Muñoz, T. Kouyama, H. Sagawa, T.M. Sato, G. Piccioni, S. Tellmann, T. Imamura, T. Satoh, Stationary waves and slowly moving features in the night upper clouds of Venus. Nat. Astron. 1, 0187 (2017a). https://doi.org/10.1038/s41550-017-0187

J. Peralta et al., Venus's winds and temperatures during the MESSENGER's flyby: an approximation to a three-dimensional instantaneous state of the atmosphere. Geophys. Res. Lett. 44, 3907-3915 (2017b). https://doi.org/10.1002/2017GL072900 
J. Peralta et al., Nightside winds at the lower clouds of Venus with Akatsuki/IR2: longitudinal, local time and decadal variations from comparison with previous measurements. Astrophys. J. Suppl. Ser. 239, 29 (2018)

A. Piccialli, D.V. Titov, D. Grassi, I. Khatuntsev, P. Drossart, G. Piccioni, A. Migliorini, Cyclostrophic winds from the Visible and Infrared Thermal Imaging Spectrometer temperature sounding: a preliminary analysis. J. Geophys. Res. 113, E00B11 (2008). https://doi.org/10.1029/2008JE003127

A. Piccialli, S. Tellmann, D.V. Titov, S.S. Limaye, I.V. Khatuntsev, M. Pätzold, B. Häusler, Dynamical properties of the Venus mesosphere from the radio-occultation experiment VeRa onboard Venus Express. Icarus 217, 669-681 (2012). https://doi.org/10.1016/j.icarus.2011.07.016

A. Piccialli, D.V. Titov, A. Sánchez-Lavega, J. Peralta, O. Shalygina, W.J. Markiewicz, H. Svedhem, High latitude gravity waves at the Venus cloud tops as observed by the Venus Monitoring Camera on board Venus Express. Icarus 227, 94-111 (2014). https://doi.org/10.1016/j.icarus.2013.09.012

J.B. Pollack, R. Young, Calculations of the radiative and dynamical state of the Venus atmosphere. J. Atmos. Sci. 32, 1025-1037 (1975)

C.C. Porco et al., Cassini imaging of Jupiter's atmosphere, satellites and rings. Science 299, 1541-1547 (2003)

S.F. Potter, G.K. Vallis, J.L. Mitchell, Spontaneous superrotation and the role of Kelvin wave-like variability in an idealized dry GCM. J. Atmos. Sci. 71, 596-614 (2014)

P. Rannou, F. Hourdin, C.P. McKay, A wind origin for Titan's haze structure. Nature 418, 853-856 (2002)

P. Rannou, F. Hourdin, C.P. McKay, D. Luz, A coupled dynamics-microphysics model of Titan's atmosphere. Icarus 170, 443-462 (2004). https://doi.org/10.1016/j.icarus.2004.03.007

P. Rannou, F. Montmessin, F. Hourdin, S. Lebonnois, The latitudinal distribution of clouds on Titan. Science 311, 201-205 (2006). https://doi.org/10.1126/science.311.5758.141c

E. Rauscher, K.M. Menou, Three-dimensional modeling of hot Jupiter atmospheric flows. Astrophys. J. 714, 1334-1342 (2010)

E. Rauscher, K.M. Menou, A general circulation model for gaseous exoplanets with double-gray radiative transfer. Astrophys. J. 750, 96 (2012)

E. Rauscher, K.M. Menou, Three-dimensional atmospheric circulation models of HD $189733 \mathrm{~b}$ and HD 209458b with consistent magnetic drag and Ohmic dissipation. Astrophys. J. 764, 103 (2013)

P.L. Read, Super-rotation and diffusion of axial angular momentum. II. A review of quasi-axisymmetric models of planetary atmospheres. Q. J. R. Meteorol. Soc. 112, 253-272 (1986). https://doi.org/10.1002/ qj. 49711247114

P.L. Read, Dynamics and circulation regimes of terrestrial planets. Planet. Space Sci. 59, 900 (2011)

P.L. Read, S. Lebonnois, Superrotation on Venus, on Titan, and elsewhere. Annu. Rev. Earth Planet. Sci. 46, 175-202 (2018). https://doi.org/10.1146/annurev-earth-082517-010137

P.B. Rhines, Waves and turbulence on a beta plane. J. Fluid Mech. 69, 417-443 (1975)

P.B. Rhines, Geostrophic turbulence. Annu. Rev. Fluid Mech. 11, 401-441 (1979)

T.M. Rogers, Constraints on the magnetic field strengths of HAT-P-7b and other hot giant exoplanets. Nat. Astron. 1, 0131 (2017)

T.M. Rogers, T.D. Komacek, Magnetic effects in hot Jupiter atmospheres. Astrophys. J. 794, 132 (2014)

T.M. Rogers, A.P. Showman, Magnetohydrodynamic simulations of the atmosphere of HD 209458b. Astrophys. J. Lett. 782, L4 (2014)

M. Roman, E. Rauscher, Modeling the effects of inhomogeneous aerosols on the hot Jupiter Kepler-7b's atmospheric circulation. Astrophys. J. 850, 17 (2017)

W.B. Rossow, A general circulation model of a Venus-like atmosphere. J. Atmos. Sci. 40, 273-302 (1983). https://doi.org/10.1175/1520-0469

W.B. Rossow, G.P. Williams, Large scale motions in the Venus stratosphere. J. Atmos. Sci. 36, 377-389 (1979)

W.B. Rossow, A.D. del Genio, T. Eichler, Cloud-tracked winds from Pioneer Venus OCPP images. J. Atmos. Sci. 47, 2053-2084 (1990). https://doi.org/10.1175/1520-0469(1990)047<2053:CTWFVO>2.0.CO;2

S. Sakai, Rossby-Kelvin instability: a new type of ageostrophic instability caused by a resonance between Rossby waves and gravity waves. J. Fluid Mech. 202, 149 (1989)

C. Salyk, A.P. Ingersoll, J. Lorre, A. Vasavada, A.D. Del Genio, Interaction between eddies and mean flow in Jupiter's atmosphere: analysis of Cassini imaging data. Icarus 185, 430-442 (2006)

A. Sánchez-Lavega, J.F. Rojas, P.V. Sada, Saturn's zonal winds at cloud level. Icarus 147, 405-420 (2000)

A. Sánchez-Lavega, S. Perez-Hoyos, J.F. Rojas, v. Hueso, R.G. French, A strong decrease in Saturn's equatorial jet at cloud level. Nature 423, 623-625 (2003)

A. Sánchez-Lavega, R. Hueso, G. Piccioni, P. Drossart, J. Peralta, S. Pérez-Hoyos, C. Wilson, F. Taylor, K. Baines, D. Luz, S. Erard, S. Lebonnois, Variable winds on Venus mapped in three dimensions. Geophys. Res. Lett. 35, L13204 (2008). https://doi.org/10.1029/2008GL033817 
A. Sánchez-Lavega, S. Lebonnois, T. Imamura, P. Read, D. Luz, The atmospheric dynamics of Venus. Space Sci. Rev. 212, 1541-1616 (2017). https://doi.org/10.1007/s11214-017-0389-x

E. Schaller et al., Storms in the tropics of Titan. Nature 460, 873-875 (2009). https://doi.org/10.1038/ nature 08193

T. Schneider, J. Liu, Formation of jets and equatorial superrotation on Jupiter. J. Atmos. Sci. 66, 579-601 (2009)

T. Schneider, S.D.B. Graves, E.L. Schaller, M.E. Brown, Polar methane accumulation and rainstorms on Titan from simulations of the methane cycle. Nature 481, 58-61 (2012). https://doi.org/10.1038/nature10666

J.T. Schofield, F.W. Taylor, Measurements of the mean, solar-fixed temperature and cloud structure of the middle atmosphere of Venus. Q. J. R. Meteorol. Soc. 109, 57-80 (1983). https://doi.org/10.1002/ qj.49710945904

J. Schou et al., Helioseismic studies of differential rotation in the solar envelope by the solar oscillations investigation using the Michelson Doppler Imager. Astrophys. J. 505, 390-417 (1998)

G. Schubert, C. Covey, A. Del Genio, L.S. Elson, G. Keating, A. Seiff, R.E. Young, J. Apt, C.C. Counselman, A.J. Kliore, S.S. Limaye, H.E. Revercomb, L.A. Sromovsky, V.E. Suomi, F. Taylor, R. Woo, U. Von Zahn, Structure and circulation of the Venus atmosphere. J. Geophys. Res. 85, 8007-8025 (1980)

R.K. Scott, L.M. Polvani, Forced-dissipative shallow-water turbulence on the sphere and the atmospheric circulation of the giant planets. J. Atmos. Sci. 64, 3158-3176 (2007)

R.K. Scott, L.M. Polvani, Equatorial superrotation in shallow atmospheres. Geophys. Res. Lett. 35, 24202 (2008)

A. Seiff, Dynamical implications of the observed thermal contrasts in Venus' upper atmosphere. Icarus 51, 574-592 (1982). https://doi.org/10.1016/0019-1035(82)90147-6

A.P. Showman, Numerical simulations of forced shallow-water turbulence: effects of moist convection on the large-scale circulation of Jupiter and Saturn. J. Atmos. Sci. 64, 3132-3157 (2007)

A.P. Showman, T.E. Dowling, Nonlinear simulations of Jupiter's 5-micron hot spots. Science 289, 1737-1740 (2000)

A.P. Showman, T. Guillot, Atmospheric circulation and tides of "51 Pegasus b-like" planets. Astron. Astrophys. 385, 166-180 (2002)

A.P. Showman, Y. Kaspi, Atmospheric dynamics of Brown dwarfs and directly imaged giant planets. Astrophys. J. 776, 85 (2013)

A.P. Showman, L.M. Polvani, The Matsuno-Gill model and equatorial superrotation. Geophys. Res. Lett. 37, L18811 (2010)

A.P. Showman, L.M. Polvani, Equatorial superrotation on tidally locked exoplanets. Astrophys. J. 738, 71 (2011)

A.P. Showman, P.J. Gierasch, Y. Lian, Deep zonal winds can result from shallow driving in a giant-planet atmosphere. Icarus 182, 513-526 (2006)

A.P. Showman et al., Atmospheric circulation of hot Jupiters: coupled radiative-dynamical general circulation model simulations of HD 189733b and HD 209458b. Astrophys. J. 699, 564-584 (2009)

A.P. Showman, J.Y-K. Cho, K. Menou, Atmospheric circulation of exoplanets, in Exoplanets, ed. by S. Seager (University of Arizona Press, Tucson, 2010), pp. 471-516

A.P. Showman, Y. Kaspi, G.R. Flierl, Scaling laws for convection and jet speeds in the giant planets. Icarus 211, 1258-1273 (2011)

A.P. Showman, J.J. Fortney, N.K. Lewis, M. Shabram, Doppler signatures of the atmospheric circulation on hot Jupiters. Astrophys. J. 762, 24 (2013a)

A.P. Showman, R.D. Wordsworth, T.M. Merlis, Y. Kaspi, Atmospheric circulation of terrestrial exoplanets, in Comparative Climatology of Terrestrial Planets, ed. by S.J. Mackwell et al. (University of Arizona Press, Tucson, 2013b), pp. 277-326

A.P. Showman, N.K. Lewis, J.J. Fortney, Three-dimensional atmospheric circulation of warm and hot Jupiters: effects of orbital distance, rotation period, and nonsynchronous rotation. Astrophys. J. 801, 95 (2015)

A.P. Showman, X. Tan, X. Zhang, Atmospheric circulation of brown dwarfs and Jupiter- and Saturn-like planets: zonal jets, long-term variability, and QBO-type oscillations. Astrophys. J. 883, 4 (2019)

B. Sicardy et al., The two Titan stellar occultations of 14 November 2003. J. Geophys. Res. 111, E11S91 (2006). https://doi.org/10.1029/2005JE002624

A.A. Simon, M.H. Wong, G.S. Orton, First results from the Hubble OPAL program: Jupiter in 2015. Astrophys. J. 812, 55 (2015). https://doi.org/10.1088/0004-637X/812/1/55

K.S. Smith, A local model for planetary atmospheres forced by small-scale convection. J. Atmos. Sci. 61, 1420-1433 (2004)

A. Spiga, S. Guerlet, E. Millour, M. Indurain, Y. Meurdesoif, S. Cabanes, T. Dubos, J. Leconte, A. Boissinot, S. Lebonnois, M. Sylvestre, T. Fouchet, Global climate modeling of Saturn's atmosphere. Part II: multiannual highresolution dynamical simulations. Icarus 335, 113377 (2020) 
N. Sugimoto, M. Takagi, Y. Matsuda, Baroclinic instability in the Venus atmosphere simulated by GCM. J. Geophys. Res., Planets 119, 1950-1968 (2014a). https://doi.org/10.1002/2014JE004624

N. Sugimoto, M. Takagi, Y. Matsuda, Waves in a Venus general circulation model. Geophys. Res. Lett. 41, 7461-7467 (2014b). https://doi.org/10.1002/2014GL061807

N. Sugimoto, A. Yamazaki, T. Kouyama, H. Kashimura, T. Enomoto, M. Takagi, Development of an ensemble Kalman filter data assimilation system for the Venusian atmosphere. Sci. Rep. 7, 9321 (2017). https://doi.org/10.1038/s41598-017-09461-1

N. Sugimoto, M. Takagi, Y. Matsuda, Fully developed superrotation driven by the mean meridional circulation in a Venus GCM. Geophys. Res. Lett. 46, 1776-1784 (2019a). https://doi.org/10.1029/2018GL080917

N. Sugimoto, T. Kouyama, M. Takagi, Impact of data assimilation on thermal tides in the case of Venus Express wind observation. Geophys. Res. Lett. 46, 4573-4580 (2019b). https://doi.org/10.1029/ 2019GL082700

H. Svedhem et al., Venus Express-the first European mission to Venus. Planet. Space Sci. 55, 1636-1652 (2007). https://doi.org/10.1016/j.pss.2007.01.013

M. Takagi, Y. Matsuda, Dynamical effect of thermal tides in the lower Venus atmosphere. Geophys. Res. Lett. 33, L13102 (2006). https://doi.org/10.1029/2006GL026168

M. Takagi, Y. Matsuda, Effects of thermal tides on the Venus atmospheric superrotation. J. Geophys. Res. 112, D09112 (2007)

M. Takagi, N. Sugimoto, H. Ando, Y. Matsuda, Three dimensional structures of thermal tides simulated by a Venus GCM. J. Geophys. Res. 123, 335-352 (2018). https://doi.org/10.1002/2017JE005449

G.I. Taylor, Experiments on the motion of solid bodies in rotating fluids. Proc. R. Soc. Lond. A 104, 213-218 (1923)

N.A. Teanby, P.G.J. Irwin, C.A. Nixon, R. de Kok, S. Vinatier, A. Coustenis, E. Sefton-Nash, S.B. Calcutt, F.M. Flasar, Active upper atmosphere chemistry and dynamics from polar circulation reversal on Titan. Nature 491, 732-735 (2012). https://doi.org/10.1038/nature11611

N.A. Teanby, B. Bézard, S. Vinatier, M. Sylvestre, C.A. Nixon, P.G.J. Irwin, R. de Kok, S.B. Calcutt, F.M. Flasar, The formation and evolution of Titan's winter polar vortex. Nat. Commun. 8, 1586 (2017). https://doi.org/10.1038/s41467-017-01839-z

N.A. Teanby, M. Sylvestre, J. Sharkey, C.A. Nixon, S. Vinatier, P.G.J. Irwin, Seasonal evolution of Titan's stratosphere during the Cassini mission. Geophys. Res. Lett. 46, 3079-3089 (2019). https:// doi.org/10.1029/2018GL081401

S. Tellmann, B. Häusle, D.P. Hinson, G.L. Tyler, T.P. Andert, M.K. Bird, T. Imamura, M. Pätzold, S. Remus, Small-scale temperature fluctuations seen by the VeRa radio science experiment on Venus Express. Icarus 221, 471-480 (2012)

T. Tokano, Mountain torque and its influence on the atmospheric angular momentum on Titan. Icarus 220, 863-876 (2012). https://doi.org/10.1016/j.icarus.2012.06.027

T. Tokano, Orbitally and geographically caused seasonal asymmetry in Titan's tropospheric climate and its implication for the lake distribution. Icarus 317, 337-353 (2019). https://doi.org/10.1016/ j.icarus.2018.07.025

T. Tokano, F.M. Neubauer, M. Laube, C.P. McKay, Seasonal variation of Titan's atmospheric structure simulated by a general circulation model. Planet. Space Sci. 47, 493-520 (1999)

T. Tokano, T. Van Hoolst, Ö. Karatekin, Polar motion of Titan forced by the atmosphere. J. Geophys. Res., Planets 116, E05002 (2011). https://doi.org/10.1029/2010JE003758

S-M. Tsai, I. Dobbs-Dixon, P-G. Gu, Three-dimensional structures of equatorial waves and the resulting super-rotation in the atmosphere of a tidally locked hot Jupiter. Astrophys. J. 793, 141 (2014)

E.P. Turtle, A.D. Del Genio, J.M. Barbara, J.E. Perry, E.L. Schaller, A.S. McEwen, R.A. West, T.L. Ray, Seasonal changes in Titan's meteorology. Geophys. Res. Lett. 38, L03203 (2011). https://doi.org/ 10.1029/2010GL046266

G.K. Vallis, M.E. Maltrud, Generation of mean flows and jets on a beta plane and over topography. J. Phys. Oceanogr. 23, 1346-1362 (1993)

A.R. Vasavada, A.P. Showman, Jovian atmospheric dynamics: an update after Galileo and Cassini. Rep. Prog. Phys. 68, 1935-1996 (2005)

S. Vinatier, B. Bézard, S. Lebonnois, N.A. Teanby, R.K. Achterberg, N. Gorius, A. Mamoutkine, E.A. Guandique, A. Jolly, E.D. Jennings, F.M. Flasar, Seasonal variations in Titan's middle atmosphere during the northern spring derived from Cassini/CIRS observations. Icarus 250, 95-115 (2015). https://doi.org/10.1016/j.icarus.2014.11.019

J.C.G. Walker, P.B. Hays, J.F. Kasting, A negative feedback mechanism for the long-term stabilization of Earth's surface temperature. J. Geophys. Res. 86, 9776-9782 (1981). https://doi.org/ 10.1029/JC086iC10p09776

P. Wang, J.L. Mitchell, Planetary ageostrophic instability leads to superrotation. Geophys. Res. Lett. 41, 4118-4126 (2014) 
M.J. Way, A.D. Del Genio, N.Y. Kiang, L.E. Sohl, D.H. Grinspoon, I. Aleinov, M. Kelley, T. Clune, Was Venus the first habitable world of our solar system? Geophys. Res. Lett. 43, 8376-8383 (2016). https://doi.org/10.1002/2016GL069790

J. Wicht, C.A. Jones, K. Zhang, Instability of zonal flows in rotating spherical shells: an application to Jupiter. Icarus 155, 425-435 (2002)

G.P. Williams, Planetary circulations: Part I: barotropic representation of the Jovian and terrestrial turbulence. J. Atmos. Sci. 35, 1399-1426 (1978)

G.P. Williams, Planetary circulations. Part II: the Jovian quasi-geostrophic regime. J. Atmos. Sci. 36, 932-968 (1979)

G.P. Williams, The dynamical range of global circulations-I. Clim. Dyn. 2, 205 (1988)

G.P. Williams, Jovian dynamics. Part III: multiple, migrating, and equatorial jets. J. Atmos. Sci. 60, 12701296 (2003)

H. Yamamoto, S. Yoden, Theoretical estimation of the superrotation strength in an idealized quasiaxisymmetric model of planetary atmospheres. J. Meteorol. Soc. Jpn. 91, 119-141 (2013). https:// doi.org/10.2151/jmsj.2013-203

M. Yamamoto, Equatorial Kelvin-like waves on slowly rotating and/or small-sized spheres: application to Venus and Titan. Icarus 322, 103-113 (2019)

M. Yamamoto, M. Takahashi, Super-rotation and equatorial waves in a T21 Venus-like AGCM. Geophys. Res. Lett. 30, 1449 (2003). https://doi.org/10.1029/2003GL016924

M. Yamamoto, M. Takahashi, Dynamics of Venus' super-rotation: the eddy momentum transport processes newly found in a GCM. Geophys. Res. Lett. 31, L09701 (2004). https://doi.org/10.1029/2004GL019518

M. Yamamoto, M. Takahashi, Dynamical effects of solar heating below the cloud layer in a Venus-like atmosphere. J. Geophys. Res. 114, E12004 (2009). https://doi.org/10.1029/2009JE003381

M. Yamamoto, K. Ikeda, M. Takahashi, T. Horinouchi, Solar-locked and geographical atmospheric structures inferred from a Venus general circulation model with radiative transfer. Icarus 321, 232-250 (2019). https://doi.org/10.1016/j.icarus.2018.11.015

J. Yang, N.B. Cowan, D.S. Abbot, Stabilizing cloud feedback dramatically expands the habitable zone of tidally locked planets. Astrophys. J. Lett. 771, L45 (2013)

J. Yang, B. Gweanel, D.C. Fabrycky, D.S. Abbot, Strong dependence of the inner edge of the habitable zone on planetary rotation rate. Astrophys. J. 787, 2 (2014)

J. Yang, D.S. Abbot, D.D. Koll, Y. Hu, A.P. Showman, Ocean dynamics and the inner edge of the habitable zone for tidally locked terrestrial planets. Astrophys. J. 871, 29 (2019)

R.E. Young, J.B. Pollack, A three-dimensional model of dynamical processes in the Venus atmosphere. J. Atmos. Sci. 34, 1315-1351 (1977)

R.M.B. Young, P.L. Read, Y. Wang, Simulating Jupiter's weather layer. Part I: jet spin-up in a dry atmosphere. Icarus 326, 225-252 (2019)

L.V. Zasova, I.V. Khatuntsev, V.M. Linkin, Thermal zonal wind in the middle atmosphere of Venus. Cosm. Res. 38, N1 (2000)

L.V. Zasova, N. Ignatiev, I. Khatuntsev, V. Linkin, Structure of the Venus atmosphere. Planet. Space Sci. 55, 1712-1728 (2007). https://doi.org/10.1016/j.pss.2007.01.011

K. Zhang, Spiralling columnar convection in rapidly rotating spherical fluid shells. J. Fluid Mech. 236, 535556 (1992)

K. Zhang, F.H. Busse, On the onset of convection in rotating spherical shells. Geophys. Astrophys. Fluid Dyn. 39, 119-147 (1987)

X. Zhang, A.P. Showman, Effects of bulk composition on the atmospheric dynamics on close-in exoplanets. Astrophys. J. 836, 73 (2017) 